\title{
A General Approximation Scheme for Solutions of Various Problems in Fixed Point Theory
}

\author{
Eric U. Ofoedu \\ Department of Mathematics, Nnamdi Azikiwe University, PMB 5025, Anambra State, Awka, Nigeria \\ Correspondence should be addressed to Eric U. Ofoedu; euofoedu@yahoo.com
}

Received 28 August 2012; Accepted 15 October 2012

Academic Editor: Antoine Ayache

Copyright (C) 2013 Eric U. Ofoedu. This is an open access article distributed under the Creative Commons Attribution License, which permits unrestricted use, distribution, and reproduction in any medium, provided the original work is properly cited.

\begin{abstract}
It is our aim to prove strong convergence of a new iterative sequence to a common element of the solution set of a generalized mixed equilibrium problem; the null space of an inverse strongly monotone operator; the set of common fixed points of a countable infinite family of nonexpansive mappings; and the set of fixed points of a continuous pseudocontractive mapping. Moreover, the common element is also a unique solution of a variational inequality problem and optimality condition for a certain minimization problem. Our theorems generalize, improve, and unify several recently announced results.
\end{abstract}

\section{Introduction}

Let $E$ be a real normed space with dual $E^{*}$. The normalized duality mapping $J$ from $E$ to $2^{E^{*}}$ is defined by

$$
J x:=\left\{x^{*} \in E^{*}:\left\langle x, x^{*}\right\rangle=\|x\|^{2},\|x\|=\left\|x^{*}\right\|\right\},
$$

where $\langle\cdot, \cdot\rangle$ denotes the generalized duality pairing. It is well known (see, e.g., [1]) that if $E^{*}$ is strictly convex, then $J$ is single-valued and if $E=H$ is a Hilbert space, then $J$ is the identity mapping. In the sequel, we shall denote the singlevalued normalized duality mapping by $j$.

A mapping $T$ with domain $D(T)$, and range $R(T)$, in $E$ is called a strict contraction or simply a contraction if and only if there exists $\gamma_{0} \in[0,1)$ such that for all $x, y \in D(T)$,

$$
\|T(x)-T(y)\| \leq \gamma_{0}\|x-y\|,
$$

and $T$ is called nonexpansive if and only if for all $x, y \in D(T)$,

$$
\|T x-T y\| \leq\|x-y\| \text {. }
$$

A point $x \in D(T)$ is called a fixed point of an operator $T$ if and only if $T x=x$. The set of fixed points of an operator $T$ is denoted by $\operatorname{Fix}(T)$, that is, $\operatorname{Fix}(T):=\{x \in D(T): T x=x\}$.

The most important generalization of the class of nonexpansive mappings is, perhaps, the class of pseudocontractive mappings. These mappings are intimately connected with the important class of nonlinear accretive operators. This connection will be made precise in what follows.

A mapping $T$ with domain $D(T)$, and range $R(T)$, in $E$ is called pseudocontractive if and only if for all $x, y \in D(T)$, the following inequality holds:

$$
\|x-y\| \leq\|(1+r)(x-y)-r(T x-T y)\|,
$$

for all $r>0$. As a consequence of a result of Kato [2], the pseudocontractive mappings can also be defined in terms of the normalized duality mappings as follows: the mapping $T$ is called pseudocontractive if and only if for all $x, y \in D(T)$, there exists $j(x-y) \in J(x-y)$ such that

$$
\langle T x-T y, j(x-y)\rangle \leq\|x-y\|^{2} .
$$

It now follows trivially from (5) that every nonexpansive mapping is pseudocontractive. We note immediately that the class of pseudocontractive mappings is larger than that of nonexpansive mappings. For examples of pseudocontractive mappings which are not nonexpansive, the reader may see [1].

To see the connection between the pseudocontractive mappings and the accretive mappings, we introduce the following definition: a mapping $A$ with domain, $D(A)$, and range, $R(A)$, in $E$ is called accretive if and only if for all $x, y \in$ $D(A)$, the following inequality is satisfied:

$$
\|x-y\| \leq\|x-y+r(A x-A y)\|,
$$


for all $r>0$. Again, as a consequence of Kato [2], it follows that $A$ is accretive if and only if for all $x, y \in D(A)$, there exists $j(x-y) \in J(x-y)$ such that

$$
\langle A x-A y, j(x-y)\rangle \geq 0 .
$$

The operator $A$ is called $\eta$-inverse strongly accretive if and only if there exists $\eta \in(0,1)$ such that for all $x, y \in D(A)$, there exists $j(x-y) \in J(x-y)$ such that

$$
\langle A x-A y, j(x-y)\rangle \geq \eta\|A x-A y\|^{2} .
$$

It is easy to see from inequalities (4) and (6) that an operator $A$ is accretive if and only if the mapping $T:=(I-A)$ is pseudocontractive. Consequently, the fixed point theory for pseudocontractive mappings is intimately connected with the mapping theory of accretive operators. For the importance of accretive operators and their connections with evolution equations, the reader may consult any of the references $[1,3]$.

Due to the above connection, fixed point theory of pseudocontractive mappings became a flourishing area of intensive research for several authors. It is of interest to note that if $E=H$ is a Hilbert space, accretive ( $\eta$-strongly accretive) operators coincide with the monotone $(\eta$-strongly monotone) operators, where an operator $A$ with domain, $D(A)$, and range, $R(A)$, in $H$ is called monotone if and only if for all $x, y \in D(A)$, we have that

$$
\langle A x-A y, x-y\rangle \geq 0
$$

and $A$ is called $\eta$-inverse strongly monotone if and only if for all $x, y \in D(A)$,

$$
\langle A x-A y, x-y\rangle \geq \eta\|A x-A y\|^{2} .
$$

A bounded linear operator $A$ with domain, $D(A)$, and range, $R(A)$, in a Hilbert space $H$ is called a strongly positive operator if and only if there exists a constant $\gamma>0$ such that for all $x \in H,\langle A x, x\rangle \geq \gamma\|x\|^{2}$.

Let $C$ be a closed convex nonempty subset of a real Hilbert space $H$ with inner product $\langle\cdot, \cdot\rangle$ and norm $\|\cdot\|$. Let $f: C \times C \rightarrow \mathbb{R}$ be a bifunction and $\Phi: C \rightarrow$ $\mathbb{R} \cup\{+\infty\}$ be a proper extended real valued function, where $\mathbb{R}$ denotes the set of real numbers. Let $\Theta: C \rightarrow H$ be a nonlinear monotone mapping. The generalized mixed equilibrium problem (GMEP) for $f$, $\Phi$, and $\Theta$ is to find $u^{*} \in$ $C$ such that

$$
\begin{aligned}
& f\left(u^{*}, y\right)+\Phi(y)-\Phi\left(u^{*}\right) \\
& \quad+\left\langle\Theta u^{*}, y-u^{*}\right\rangle \geq 0 \quad \forall y \in C .
\end{aligned}
$$

The set of solutions for GMEP (11) is denoted by

$$
\begin{aligned}
\operatorname{GMEP}(f, \Phi, \Theta)=\{u & \in C: f(u, y)+\Phi(y)-\Phi(u) \\
& +\langle\Theta u, y-u\rangle \geq 0 \forall y \in C\} .
\end{aligned}
$$

If $\Phi \equiv 0 \equiv \Theta$ in (11), then (11) reduces to the classical equilibrium problem (EP), that is, the problem of finding $u^{*} \in$ $C$ such that

$$
f\left(u^{*}, y\right) \geq 0 \quad \forall y \in C,
$$

and $\operatorname{GMEP}(f, 0,0)$ is denoted by $\operatorname{EP}(f)$, where

$$
\mathrm{EP}(f)=\{u \in C: f(u, y) \geq 0 \forall y \in C\} .
$$

If $f \equiv 0 \equiv \Phi$ in (11), then GMEP (11) reduces to the classical variational inequality problem and $\operatorname{GMEP}(0,0, \Theta)$ is denoted by $\operatorname{VI}(\Theta, C)$, where

$$
\mathrm{VI}(\Theta, C)=\{u \in C:\langle\Theta u, y-u\rangle \geq 0 \forall y \in C\} .
$$

If $f \equiv 0 \equiv \Theta$, then GMEP (11) reduces to the following minimization problem:

$$
\text { find } u^{*} \in C \text { such that } \Phi(y) \geq \Phi\left(u^{*}\right) \quad \forall y \in C \text {, }
$$

and $\operatorname{GMEP}(0, \Phi, 0)$ is denoted by $\operatorname{Argmin}(\Phi)$, where

$$
\operatorname{Argmin}(\Phi)=\{u \in C: \Phi(u) \leq \Phi(y) \forall y \in C\} .
$$

If $\Theta \equiv 0$, then (11) becomes the mixed equilibrium problem (MEP) and $\operatorname{GMEP}(f, \Phi, 0)$ is denoted by $\operatorname{MEP}(f, \Phi)$, where

$$
\begin{aligned}
& \operatorname{MEP}(f, \Phi) \\
& \quad=\{u \in C: f(u, y)+\Phi(y)-\Phi(u) \geq 0 \forall y \in C\}
\end{aligned}
$$

If $\Phi \equiv 0$, then (11) reduces to the generalized equilibrium problem $(\mathrm{GEP})$ and $\operatorname{GMEP}(f, 0, \Theta)$ is denoted by $\operatorname{GEP}(f, \Theta)$, where

$$
\begin{aligned}
& \operatorname{GEP}(f, \Theta) \\
& \quad=\{u \in C: f(u, y)+\langle\Theta u, y-u\rangle \geq 0 \forall y \in C\} .
\end{aligned}
$$

If $f \equiv 0$, then GMEP (11) reduces to the generalized variational inequality problem (abbreviated GVIP) and $\operatorname{GMEP}(0, \Phi, \Theta)$ is denoted by $\operatorname{GVI}(\Phi, \Theta, C)$, where

$$
\begin{aligned}
\operatorname{GVI} & (\Phi, \Theta, C) \\
& =\{u \in K: \Phi(y)-\Phi(u)+\langle\Theta u, y-u\rangle \geq 0 \forall y \in C\}
\end{aligned}
$$

The generalized mixed equilibrium problem (GMEP) includes special cases as the monotone inclusion problems, saddle point problems, variational inequality problems, minimization problems, optimization problems, vector equilibrium problems, and Nash equilibria in noncooperative games. Furthermore, there are several other problems, for example, the complementarity problems and fixed point problems, which can also be written in the form of the generalized mixed equilibrium problem. In other words, the generalized mixed equilibrium problem is a unifying model for several problems arising from engineering, physics, statistics, computer science, optimization theory, operations research, economics, and countless other fields. For the past 20 years or so, many existence results have been published for various equilibrium problems (see, e.g., [4-6]).

Iterative approximation of fixed points and zeros of nonlinear operators has been studied extensively by many authors to solve nonlinear operator equations as well as variational inequality problems and their generalizations (see, e.g., [724]). Most published results on nonexpansive mappings (e.g.) 
focus on the iterative approximation of their fixed points or approximation of common fixed points of a given family of this class of mappings.

Let $C$ be a closed convex nonempty subset of $H$, let $T$ : $C \rightarrow C$ be a nonexpansive mapping such that $\operatorname{Fix}(T) \neq \varnothing$. Given that $u \in C$ and a real sequence $\left\{\alpha_{n}\right\}_{n \geq 0}$ in the interval $(0,1)$, let a sequence $\left\{x_{n}\right\}_{n \geq 0}$ be defined by

$$
x_{0} \in C, \quad x_{n+1}=\alpha_{n} u+\left(1-\alpha_{n}\right) T x_{n}, \quad n \geq 0 .
$$

Under appropriate conditions on the iterative parameter $\left\{\alpha_{n}\right\}_{n \geq 0}$, it was shown by Bauschke [25], Halpern [26], Lions [27], and Wittmann [28] that $\left\{x_{n}\right\}_{n \geq 0}$ converges strongly to $P_{F(T)} u$, the projection of $u$ to the fixed point set, $F(T)$, of $T$.

Marino and $\mathrm{Xu}[11]$, proved that the iteration scheme given by

$$
x_{0} \in H, \quad x_{n+1}=\alpha_{n} \gamma f\left(x_{n}\right)+\left(I-\alpha_{n} A\right) T x_{n}, \quad n \geq 0
$$

converges strongly to a unique solution $x^{\prime} \in \operatorname{Fix}(T)$ of the variational inequality

$$
\left\langle(\gamma f-A) x^{\prime}, y-x^{\prime}\right\rangle \leq 0 \quad \forall y \in \operatorname{Fix}(T),
$$

which is the optimality condition for the minimization problem

$$
\min _{x \in \operatorname{Fix}(T)}\left(\frac{1}{2}\langle A x, x\rangle-h(c)\right),
$$

where $h$ is a potential function for $\gamma f$ (i.e., $h^{\prime}(x)=\gamma f(x)$ for all $x \in H$ ); provided $f: H \rightarrow H$ is a strict contraction, $T: H \rightarrow H$ is nonexpansive and the iterative parameter $\left\{\alpha_{n}\right\}_{n \geq 0}$ that satisfies appropriate conditions.

In [29], Yamada introduced the following hybrid iterative method:

$$
x_{0} \in H, \quad x_{n+1}=T x_{n}-\mu \lambda_{n} A\left(T x_{n}\right), \quad n \geq 0,
$$

where $T$ is nonexpansive, $A$ is $L$-Lipschitzian and an $\eta$ strongly monotone operator. He proved that if $\mu$ and $\left\{\lambda_{n}\right\}_{n \geq 1}$ satisfy appropriate conditions, then (25) converges strongly to a unique solution $x^{\prime} \in \operatorname{Fix}(T)$ of the varational inequality $\left\langle A x^{\prime}, y-x^{\prime}\right\rangle \geq 0$, for all $y \in F(T)$.

Tian [30] introduced the following iterative method:

$$
x_{0} \in H, \quad x_{n+1}=\alpha_{n} \gamma f\left(x_{n}\right)+\left(I-\mu \alpha_{n} A\right) T x_{n}, \quad n \geq 0,
$$

and proved that if $f: H \rightarrow H$ is a contraction, $A: H \rightarrow$ $H$ is an $\eta$-strongly monotone mapping, $T: H \rightarrow H$ a nonexpansive mapping and the parameter $\left\{\alpha_{n}\right\}_{n \geq 1}$ satisfies appropriate conditions, then the sequence $\left\{x_{n}\right\}_{n \geq 1}$ converges strongly to a unique solution $x^{\prime} \in \operatorname{Fix}(T)$ of the variational inequality

$$
\left\langle(\gamma f-\mu A) x^{\prime}, y-x^{\prime}\right\rangle \leq 0 \quad \forall y \in \operatorname{Fix}(T) .
$$

Let $C$ be a convex nonempty subset of a real Banach space. Let $\left\{T_{j}\right\}_{j \geq 1}$ be a countable infinite family of nonexpansive mappings of $C$ into itself and let $\left\{\lambda_{j}\right\}_{j \geq 1}$ be a sequence of real numbers such that $\lambda_{j} \in(0,1)$ for all $j \in \mathbb{N}$. For all $n \in \mathbb{N}$, define a mapping $W_{n}: C \rightarrow C$ by

$$
\begin{aligned}
U_{n, n+1} & :=I, \\
U_{n, n} & :=\lambda_{n} T_{n} U_{n, n+1}+\left(1-\lambda_{n}\right) I, \\
U_{n, n-1} & :=\lambda_{n-1} T_{n-1} U_{n, n}+\left(1-\lambda_{n-1}\right) I, \\
& \vdots \\
U_{n, k} & :=\lambda_{k} T_{k} U_{n, k+1}+\left(1-\lambda_{k}\right) I, \\
U_{n, k-1}: & \lambda_{k-1} T_{k-1} U_{n, k}+\left(1-\lambda_{k-1}\right) I, \\
& \vdots \\
U_{n, 2} & :=\lambda_{2} T_{2} U_{n, 3}+\left(1-\lambda_{2}\right) I, \\
W_{n} & :=U_{n, 1}=\lambda_{1} T_{1} U_{n, 2}+\left(1-\lambda_{1}\right) I .
\end{aligned}
$$

The mapping $W_{n}, n \in \mathbb{N}$ is called the $W$-mapping generated by the countable infinite family $\left\{T_{j}\right\}_{j \geq 1}$ of nonexpansive mappings (see, e.g., [31]).

In [32], Yao et al. studied the following problem. Let $H$ be a real Hilbert space. Consider the iterative scheme

$$
\begin{aligned}
x_{1} \in H, \quad x_{n+1}= & \alpha_{n} \gamma f\left(x_{n}\right) \\
& +\beta_{n} x_{n}+\left(\left(1-\beta_{n}\right) I-\alpha_{n} A\right) W_{n} x_{n},
\end{aligned}
$$

where $\gamma>0$ is some constant, $f: H \rightarrow H$ is a given contractive mapping, and $A$ is a strongly positive bounded linear operator on $H$. Assuming that $F:=\bigcap_{j=1}^{\infty} \operatorname{Fix}\left(T_{j}\right) \neq \varnothing$, and under appropriate conditions on the iterative parameters, Yao et al. proved that the sequence $\left\{x_{n}\right\}_{n \geq 1}$ generated by (29) converges strongly to $x^{*} \in F$, where $x^{*}$ is a unique solution of the variational inequality

$$
\left\langle(A-\gamma f) x^{*}, y-x^{*}\right\rangle \geq 0 \quad \forall y \in F .
$$

Colao and Marino [33] proved that if $\left\{x_{n}\right\}_{n \geq 1}$ and $\left\{u_{n}\right\}_{n \geq 1}$ are sequences generated by $x_{1} \in H$ and

$$
\begin{aligned}
G\left(u_{n}, y\right) & +\frac{1}{r_{n}}\left\langle y-u_{n}, u_{n}-x_{n}\right\rangle \geq 0 \quad y \in H ; \\
x_{n+1}= & \varepsilon_{n} \gamma f\left(x_{n}\right)+\beta_{n} x_{n} \\
& +\left(\left(1-\beta_{n}\right) I-\varepsilon_{n} A\right) W_{n} u_{n}, \quad n \geq 1,
\end{aligned}
$$

(where $\varepsilon_{n}, \beta_{n}, r_{n}$, and $\gamma$ satisfy appropriate conditions), then both $\left\{x_{n}\right\}_{n \geq 1}$ and $\left\{u_{n}\right\}_{n \geq 1}$ converge strongly to an element $x^{*} \epsilon$ $\bigcap_{j=1}^{\infty} \operatorname{Fix}\left(T_{j}\right) \cap \operatorname{EP}(G)$, which is also a unique solution of the variational inequality

$$
\left\langle(A-\gamma f) x^{*}, y-x^{*}\right\rangle \geq 0 \quad \forall y \in \bigcap_{j=1}^{\infty} \operatorname{Fix}\left(T_{j}\right) \cap \operatorname{EP}(G) .
$$


Let $C$ be a nonempty closed and convex subset of real Hilbert space $H$. Let $T: C \rightarrow C$ be a continuous pseudocontractive mapping; $A: C \rightarrow H$ be a continuous monotone mapping; $B: H \rightarrow H$ an inverse strongly monoyone mapping. For each $n \in \mathbb{N}$, let $T_{r_{n}}$ and $F_{r_{n}}$ be defined for each $x \in H$ by

$$
\begin{aligned}
& T_{r_{n}}:=\{z \in C:\langle y-z, T z\rangle \\
&\left.\quad-\frac{1}{r_{n}}\left\langle y-z,\left(1+r_{n}\right) z-x\right\rangle \leq 0, \forall y \in C\right\}, \\
& F_{r_{n}}:=\{z \in C:\langle y-z, A z\rangle \\
&\left.\quad+\frac{1}{r_{n}}\langle y-z, z-x\rangle \leq 0, \forall y \in C\right\} .
\end{aligned}
$$

Let $\left\{x_{n}\right\}_{n \geq 1}$ be a sequence generated iteratively by $x_{1} \in C$,

$$
\begin{aligned}
y_{n}= & F_{r_{n}} x_{n} ; \\
x_{n+1}= & \alpha_{n} \gamma f\left(x_{n}\right)+\delta_{n} x_{n} \\
& +\left(\left(1-\delta_{n}\right) I-\alpha_{n} B\right) T_{r_{n}} y_{n}, \quad n \geq 1,
\end{aligned}
$$

(where $\alpha_{n}, \delta_{n}, r_{n}$, and $\gamma$ satisfy appropriate conditions), then it was recently proved by Chamnarnpan and Kumam [34] that the sequence $\left\{x_{n}\right\}_{n \geq 1}$ defined by (34) converges strongly to an element $z \in \operatorname{Fix}(T) \cap \operatorname{VI}(C, A)$, which is a unique solution of the variational inequality

$$
\langle(B-\gamma f) z, x-z\rangle \geq 0 \quad \forall x \in \operatorname{Fix}(T) \cap \operatorname{VI}(C, A) .
$$

In this paper, motivated by the results of the authors mentioned above, it is our aim to prove strong convergence of a new iterative sequence to a common element of the solution set of a generalized mixed equilibrium problem; the null space of an inverse strongly monotone operator; the set of common fixed points of a countable infinite family of nonexpansive mappings; the set of fixed points of a continuous pseudocontractive mapping. Moreover, the element is also a unique solution of a variational inequality problem and optimality condition for a certain minimization problem. Our theorems generalize improve and unify several recently announced results.

\section{Preliminary}

In what follows, we shall make use of the following lemmas.

Lemma 1 (see, e.g., [31]). Let $C$ be a closed convex nonempty subset of a strictly convex real Banach space. Let $\left\{T_{j}\right\}_{j \geq 1}$ be a countable infinite family of nonexpansive mappings of $C$ into itself and let $\left\{\lambda_{j}\right\}_{j \geq 1}$ be a real sequence such that $0<\lambda_{j} \leq b<1$ for all $j \in \mathbb{N}$, for some constant $b \in(0,1)$. Then, for all $x \in C$ and $k \in \mathbb{N}, \lim _{n \rightarrow \infty} U_{n, k} x$ exists (where $U_{n, k}$ is as defined in (28)).

In particular, for $k=1$ in Lemma 1 , we define a mapping $W: C \rightarrow C$ by

$$
W x:=\lim _{n \rightarrow \infty} U_{n, 1} x=\lim _{n \rightarrow \infty} W_{n} x .
$$

Lemma 2 (see, e.g., [31]). Let $C$ be a closed convex nonempty subset of a strictly convex real Banach space. Let $\left\{T_{j}\right\}_{j \geq 1}$ be a countable infinite family of nonexpansive mappings of $C$ into itself such that $\bigcap_{j=1}^{\infty} \operatorname{Fix}\left(T_{j}\right) \neq \varnothing$ and let $\left\{\lambda_{j}\right\}_{j \geq 1}$ be a real sequence such that $0<\lambda_{j} \leq b<1$ for all $j \in \mathbb{N}$, for some constant $b \in(0,1)$. Then, $\operatorname{Fix}(W)=\bigcap_{j=1}^{\infty} \operatorname{Fix}\left(T_{j}\right)=$ $\bigcap_{n=1}^{\infty} \operatorname{Fix}\left(W_{n}\right)$.

Lemma 3 (see [33]). Let $C$ be a closed convex nonempty subset of a strictly convex real Banach space. Let $\left\{T_{j}\right\}_{j \geq 1}$ be a countable infinite family of nonexpansive mappings of $C$ into itself such that $\bigcap_{j=1}^{\infty} \operatorname{Fix}\left(T_{j}\right) \neq \varnothing$ and let $\left\{\lambda_{j}\right\}_{j \geq 1}$ be a real sequence such that $0<\lambda_{j} \leq b<1$ for all $j \in \mathbb{N}$, for some constant $b \in(0,1)$. Suppose that $W_{n}: C \rightarrow C$ is given by (28) for all $n \in \mathbb{N}$, then

$$
\begin{aligned}
& \left\|W_{n+1} x-W_{n} x\right\| \\
& \quad \leq 2 \prod_{j=1}^{n} \lambda_{j}\|x-p\|, \quad \forall x \in C, \quad p \in \bigcap_{j=1}^{\infty} \operatorname{Fix}\left(T_{j}\right) .
\end{aligned}
$$

Lemma 4. Let $H$ be a real Hilbert space, then the following inequality holds:

$$
\|x+y\|^{2} \leq\|x\|^{2}+2\langle y, x+y\rangle \quad \forall x, y \in H .
$$

Lemma 5 (see, e.g., [35-37]). Let $\left\{\lambda_{n}\right\}_{n \geq 0}$ be a sequence of nonnegative real numbers satisfying the following condition:

$$
\lambda_{n+1} \leq\left(1-\alpha_{n}\right) \lambda_{n}+\sigma_{n}, \quad n \geq 0,
$$

where $\left\{\alpha_{n}\right\}_{n \geq 0}$ and $\left\{\sigma_{n}\right\}_{n \geq 0}$ are sequences of real numbers such that $\left\{\alpha_{n}\right\}_{n \geq 0} \subset[0,1], \sum_{n=0}^{\infty} \alpha_{n}=+\infty$. Suppose that $\sigma_{n}=$ $o\left(\alpha_{n}\right), n \geq 0$ (i.e., $\lim _{n \rightarrow \infty}\left(\sigma_{n} / \alpha_{n}\right)=0$ ) or $\sum_{n=0}^{\infty}\left|\sigma_{n}\right|<+\infty$ or $\lim \sup _{n \rightarrow \infty}\left(\sigma_{n} / \alpha_{n}\right) \leq 0$, then $\lambda_{n} \rightarrow 0$ as $n \rightarrow \infty$.

Lemma 6 (see, e.g., Suzuki [38]). Let $\left\{t_{n}\right\}_{n \geq 0}$ and $\left\{y_{n}\right\}_{n \geq 0}$ be two bounded sequences in a real Banach space such that $y_{n+1}=\beta_{n} y_{n}+\left(1-\beta_{n}\right) t_{n}$, for all $n \geq 0$, where $\left\{\beta_{n}\right\}_{n \geq 0}$ is a real sequence satisfying the condition $0<\liminf _{n \rightarrow \infty} \beta_{n} \leq$ $\lim \sup _{n \rightarrow \infty} \beta_{n}<1$. Suppose that

$$
\limsup _{n \rightarrow \infty}\left(\left\|t_{n+1}-t_{n}\right\|-\left\|y_{n+1}-y_{n}\right\|\right) \leq 0,
$$

then $\lim _{n \rightarrow \infty}\left\|t_{n}-y_{n}\right\|=0$.

Lemma 7 (see Zegeye [39]). Let $C$ be a nonempty closed convex subset of a real Hilbert space $H$. Let $T^{\prime}: C \rightarrow H$ be a continuous pseudocontractive mapping, then for all $r>0$ and $x \in H$, there exists $z \in C$ such that

$$
\left\langle y-z, T^{\prime} z\right\rangle-\frac{1}{r}\langle y-z,(1+r) z-x\rangle \leq 0 \quad \forall y \in C .
$$

Lemma 8 (see Zegeye [39]). Let $C$ be a nonempty closed convex subset of a real Hilbert space $H$. Let $T^{\prime}: C \rightarrow C$ be a continuous pseudocontractive mapping, then for all $r>0$ and $x \in H$, define a mapping $F_{r}: H \rightarrow C$ by

$$
\begin{aligned}
F_{r} x= & \left\{z \in C:\left\langle y-z, T^{\prime} z\right\rangle\right. \\
& \left.\quad-\frac{1}{r}\langle y-z,(1+r) z-x\rangle \leq 0 \forall y \in C\right\},
\end{aligned}
$$


then the following hold:

(1) $F_{r}$ is single-valued;

(2) $F_{r}$ is firmly nonexpansive type mapping, that is, for all $x, y \in H$,

$$
\left\|F_{r} x-F_{r} y\right\|^{2} \leq\left\langle F_{r} x-F_{r} y, x-y\right\rangle ;
$$

(3) $\operatorname{Fix}\left(F_{r}\right)$ is closed and convex; and $\operatorname{Fix}\left(F_{r}\right)=\operatorname{Fix}\left(T^{\prime}\right)$ for all $r>0$.

In the sequel, we shall require that the bifunction $f: C \times$ $C \rightarrow \mathbb{R}$ satisfies the following conditions:

(A1) $f(x, x)=0$ for all $x \in C$;

(A2) $f$ is monotone, in the sense that $f(x, y)+f(y, x) \leq 0$ for all $x, y \in C$;

(A3) $\lim \sup _{t \rightarrow 0^{+}} f(t z+(1-t) x, y) \leq f(x, y)$ for all $x, y, z \in$ C;

(A4) the function $y \mapsto f(x, y)$ is convex and lower semicontinuous for all $x \in C$.

Lemma 9 (Compare with Lemma 2.4 of [5]). Let $C$ be a closed convex nonempty subset of a real Hilbert space $H$. Let $f: C \times$ $C \rightarrow \mathbb{R}$ be a bifunction satisfying conditions $(A 1)-(A 4) ; \Theta$ : $C \rightarrow H$ a continuous monotone mapping and $\Phi: C \rightarrow \mathbb{R} \cup$ $\{+\infty\}$ a proper lower semicontinuous convex function. Then, for all $r>0$ and $x \in H$ there exists $u \in C$ such that

$$
\begin{array}{r}
f(u, y)+\Phi(y)-\Phi(u)+\langle\Theta u, y-u\rangle \\
+\frac{1}{r}\langle y-u, u-x\rangle \geq 0 \quad \forall y \in K .
\end{array}
$$

Moreover, if for all $x \in H$ we define a mapping $G_{r}: H \rightarrow 2^{C}$ by

$$
\begin{aligned}
G_{r}(x)=\{u & \in C: f(u, y)+\Phi(y)-\Phi(u) \\
& +\langle\Theta u, y-u\rangle+\frac{1}{r}\langle y-u, u-x\rangle \\
& \geq 0, \forall y \in C\},
\end{aligned}
$$

then the following hold:

(1) $G_{r}$ is single-valued for all $r>0$;

(2) $G_{r}$ is firmly nonexpansive, that is, for all $x, z \in H$,

$$
\left\|G_{r} x-G_{r} z\right\|^{2} \leq\left\langle G_{r} x-G_{r} z, x-z\right\rangle ;
$$

(3) $\operatorname{Fix}\left(G_{r}\right)=\operatorname{GMEP}(f, \Phi, \Theta)$ for all $r>0$;

(4) $\operatorname{GMEP}(f, \Phi, \Theta)$ is closed and convex.

Let $C$ be a closed convex nonempty subset of a uniformly convex real Banach space $E$, where $E$ is called uniformly convex if and only if the modulus of convexity

$$
\delta_{E}(\varepsilon)=\inf \left\{1-\frac{1}{2}\|x+y\|:\|x\| \leq 1,\|y\| \leq 1,\|x-y\| \geq \varepsilon\right\}
$$

is positive for all $\varepsilon \in(0,2]$. Given a bounded sequence $\left\{x_{n}\right\}_{n \geq 1}$ in $C$, define

$$
r_{m}(y):=\sup \left\{\left\|x_{k}-y\right\|: k \geq m\right\}, \quad y \in E .
$$

It is well known (see, e.g., [40]) that there exists unique $c_{m} \in C$ such that

$$
r_{m}\left(c_{m}\right)=\inf \left\{r_{m}(y): y \in C\right\}=r_{m} \quad \forall m \in \mathbb{N} .
$$

Clearly, $r_{m+1} \leq r_{m}$ and $0 \leq r_{m}$ for all $m \in \mathbb{N}$, so that $\lim _{m \rightarrow \infty} r_{m}=\inf _{m \in \mathbb{N}} r_{m}$ exists. It is known (see [40]) that if $\lim _{m \rightarrow \infty} r_{m}=\inf _{m \in \mathbb{N}} r_{m}=0$, then the sequence $\left\{x_{n}\right\}_{n \geq 1}$ converges.

Definition 10 (see [40]). If the sequence $\left\{c_{m}\right\}_{m \geq 1}$ converges, then $c_{0}=\lim _{m \rightarrow \infty} c_{m}$ is called the asymptotic center of $\left\{x_{n}\right\}_{n \geq 1}$ (with respect to $C$ ). Equivalently (see, e.g., [22]), $c_{0} \in C$ is called the asymptotic center of $\left\{x_{n}\right\}_{n \geq 1}$ (with respect to $C$ ) if and only if

$$
\limsup _{n \rightarrow \infty}\left\|x_{n}-c_{0}\right\|=\inf _{z \in C} \limsup _{n \rightarrow \infty}\left\|x_{n}-z\right\| .
$$

Lemma 11 (see Theorem 1 of Edelstein [40]). Let $C$ be $a$ closed convex nonempty subset of a uniformly convex real Banach space $E$, Let $\left\{x_{n}\right\}_{n \geq 1}$ be a bounded sequence in $C$ and $\left\{c_{m}\right\}_{m \geq 1}$ be as in (49), then $c=\lim _{m \rightarrow \infty} c_{m}$ exists. In other words, the asymptotic center $c$ of the sequence $\left\{x_{n}\right\}_{n \geq 1}$ (with respect to $C$ ) exists and is unique.

\section{Main Results}

Lemma 12. Let $C$ be a nonempty closed convex subset of a real Hilbert space $H$. Let $T^{\prime}: C \rightarrow C$ be a continuous pseudocontractive mapping. For $r>0$, let $F_{r}: H \rightarrow C$ be the mapping in Lemma 8, then for any $x \in H$ and for any $p, q>0$,

$$
\left\|F_{p} x-F_{q} x\right\| \leq \frac{|p-q|}{p}\left(\left\|F_{p} x\right\|+\|x\|\right) .
$$

Proof. Observe that for any $y \in C$,

$$
\begin{aligned}
& \left\langle y-F_{p} x, T^{\prime} F_{p} x\right\rangle-\frac{1}{p}\left\langle y-F_{p} x,(1+p) F_{p} x-x\right\rangle \leq 0, \\
& \left\langle y-F_{q} x, T^{\prime} F_{q} x\right\rangle-\frac{1}{q}\left\langle y-F_{q} x,(1+q) F_{q} x-x\right\rangle \leq 0 .
\end{aligned}
$$

So, we obtain (in particular) for $y=F_{q} x$ in (52) and $y=F_{p} x$ in (53) that

$$
\begin{aligned}
\left\langle F_{q} x-F_{p} x, T^{\prime} F_{p} x\right\rangle & \\
- & \frac{1}{p}\left\langle F_{q} x-F_{p} x,(1+p) F_{p} x-x\right\rangle \leq 0, \\
\left\langle F_{p} x-F_{q} x, T^{\prime} F_{q} x\right\rangle & -\frac{1}{q}\left\langle F_{p} x-F_{q} x,(1+q) F_{q} x-x\right\rangle \leq 0,
\end{aligned}
$$


respectively. Adding (54) and (55) gives

$$
\begin{aligned}
& -\left\langle F_{q} x-F_{p} x, T^{\prime} F_{q} x-T^{\prime} F_{p} x\right\rangle \\
& -\left\langle F_{q} x-F_{p} x, \frac{(1+p) F_{p} x-x}{p}-\frac{(1+q) F_{q} x-x}{q}\right\rangle \leq 0 .
\end{aligned}
$$

Using the fact that $T^{\prime}$ is pseudocontractive, we obtain from (56) that

$$
\begin{aligned}
- & \left\langle F_{q} x-F_{p} x, F_{q} x-F_{p} x\right\rangle \\
& -\left\langle F_{q} x-F_{p} x, \frac{(1+p) F_{p} x-x}{p}-\frac{(1+q) F_{q} x-x}{q}\right\rangle \leq 0 .
\end{aligned}
$$

This implies that

$$
\begin{aligned}
& \left\langle F_{q} x-F_{p} x, F_{q} x-F_{p} x\right\rangle \\
& +\left\langle F_{q} x-F_{p} x, \frac{(1+p) F_{p} x-x}{p}-\frac{(1+q) F_{q} x-x}{q}\right\rangle \geq 0 .
\end{aligned}
$$

Equivalently, we obtain

$$
\left\langle F_{q} x-F_{p} x, \frac{F_{p} x-x}{p}-\frac{F_{q} x-x}{q}\right\rangle \geq 0 .
$$

This implies that

$$
\begin{aligned}
0 \leq & \left\langle F_{p} x-F_{q} x, F_{q} x-x-\frac{q}{p}\left(F_{p} x-x\right)\right\rangle \\
& =\left\langle F_{p} x-F_{q} x, F_{q} x-F_{p} x+F_{p} x-x-\frac{q}{p}\left(F_{p} x-x\right)\right\rangle \\
= & \left\langle F_{p} x-F_{q} x, F_{q} x-F_{p} x+\left(1-\frac{q}{p}\right)\left(F_{p} x-x\right)\right\rangle \\
\leq & -\left\|F_{p} x-F_{q} x\right\|^{2}+\left|1-\frac{q}{p}\right|\left\|F_{p} x-F_{q} x\right\| \\
& \times\left(\left\|F_{p} x\right\|+\|x\|\right) .
\end{aligned}
$$

Thus,

$$
\left\|F_{p} x-F_{q} x\right\| \leq \frac{|p-q|}{p}\left(\left\|F_{p} x\right\|+\|x\|\right) .
$$

Lemma 13. Let $C$ be a closed convex nonempty subset of a real Hilbert space $H$. Let $f: C \times C \rightarrow \mathbb{R}$ be a bifunction satisfying conditions $(A 1)-(A 4) ; \Theta: C \rightarrow H$ a monotone mapping and $\Phi: C \rightarrow \mathbb{R} \cup\{+\infty\}$ a proper lower semicontinuous convex function. Let $r>0$ and let $G_{r}$ be the mapping in Lemma 9 , then for all $p, q>0$ and for all $x \in H$, one has that

$$
\left\|G_{p} x-G_{q} x\right\| \leq \frac{|p-q|}{p}\left(\left\|G_{p} x\right\|+\|x\|\right) .
$$

Proof. We observe that

$$
\begin{gathered}
f\left(G_{p} x, y\right)+\Phi(y)-\Phi\left(G_{p} x\right)+\left\langle\Theta\left(G_{p} x\right), y-G_{p} x\right\rangle \\
+\frac{1}{p}\left\langle y-G_{p} x, G_{p} x-x\right\rangle \geq 0 \quad \forall y \in H, \\
f\left(G_{q} x, y\right)+\Phi(y)-\Phi\left(G_{q} x\right)+\left\langle\Theta\left(G_{q} x\right), y-G_{q} x\right\rangle \\
+\frac{1}{q}\left\langle y-G_{q} x, G_{q} x-x\right\rangle \geq 0 \quad \forall y \in H .
\end{gathered}
$$

In particular, we obtain from (63) and (64) (resp.) that

$$
\begin{aligned}
f\left(G_{p} x, G_{q} x\right)+\Phi\left(G_{q} x\right)-\Phi\left(G_{p} x\right) \\
+\left\langle\Theta\left(G_{p} x\right), G_{q} x-G_{p} x\right\rangle \\
+\frac{1}{p}\left\langle G_{q} x-G_{p} x, G_{p} x-x\right\rangle \geq 0, \\
f\left(G_{q} x, G_{p} x\right)+\Phi\left(G_{p} x\right)-\Phi\left(G_{q} x\right) \\
+\left\langle\Theta\left(G_{q} x\right), G_{p} x-G_{q} x\right\rangle \\
+\frac{1}{q}\left\langle G_{p} x-G_{q} x, G_{q} x-x\right\rangle \geq 0 .
\end{aligned}
$$

Adding (65) (and using the monotonicity of $f$ and $\Theta$ ), we get that

$$
0 \leq\left\langle G_{p} x-G_{q} x, \frac{G_{q} x-x}{q}-\frac{G_{p} x-x}{p}\right\rangle .
$$

This implies that

$$
\begin{aligned}
0 \leq & \left\langle G_{p} x-G_{q} x, G_{q} x-x-\frac{q}{p}\left(G_{p} x-x\right)\right\rangle \\
= & \left\langle G_{p} x-G_{q} x, G_{q} x-G_{p} x+G_{p} x-x-\frac{q}{p}\left(G_{p} x-x\right)\right\rangle \\
= & \left\langle G_{p} x-G_{q} x, G_{q} x-G_{p} x+\left(1-\frac{q}{p}\right)\left(G_{p} x-x\right)\right\rangle \\
\leq & -\left\|G_{p} x-G_{q} x\right\|^{2}+\left|1-\frac{q}{p}\right| \\
& \times\left\|G_{p} x-G_{q} x\right\|\left(\left\|G_{p} x\right\|+\|x\|\right) .
\end{aligned}
$$

Thus,

$$
\left\|G_{p} x-G_{q} x\right\| \leq \frac{|p-q|}{p}\left(\left\|G_{p} x\right\|+\|x\|\right) .
$$

Lemma 14. Let $\left\{a_{n}\right\}_{n \geq 1}$ be a bounded sequence of nonnegative real numbers, then

$$
\limsup _{n \rightarrow \infty} a_{n}^{2}=\left(\limsup _{n \rightarrow \infty} a_{n}\right)^{2} .
$$


Proof. It is well known that for any two bounded sequences $\left\{u_{n}\right\}_{n \geq 1}$ and $\left\{v_{n}\right\}_{n \geq 1}$ of nonnegative real numbers, we have that

$$
\limsup _{n \rightarrow \infty} u_{n} v_{n} \leq \limsup _{n \rightarrow \infty} u_{n} \times \limsup _{n \rightarrow \infty} v_{n} \text {. }
$$

It then follows that for any bounded sequence $\left\{a_{n}\right\}_{n \geq 1}$ of nonnegative terms,

$$
\limsup _{n \rightarrow \infty} a_{n}^{2} \leq\left(\limsup _{n \rightarrow \infty} a_{n}\right)^{2} \text {. }
$$

To complete our proof, we show that

$$
\limsup _{n \rightarrow \infty} a_{n}^{2} \geq\left(\limsup _{n \rightarrow \infty} a_{n}\right)^{2} .
$$

Now, let $\left\{a_{n_{j}}\right\}_{j \geq 1}$ be a subsequence of $\left\{a_{n}\right\}_{n \geq 1}$ such that $\lim \sup _{n \rightarrow \infty} a_{n}=\lim _{j \rightarrow \infty} a_{n_{j}}$, then by the continuity of the function $f: \mathbb{R} \rightarrow \mathbb{R}$ given by $f(t)=t^{2}$, we obtain that

$$
\left(\limsup _{n \rightarrow \infty} a_{n}\right)^{2}=\left(\lim _{j \rightarrow \infty} a_{n_{j}}\right)^{2}=\lim _{j \rightarrow \infty} a_{n_{j}}^{2} \leq \limsup _{n \rightarrow \infty} a_{n}^{2} \text {. }
$$

The last inequality follows from the fact that $\limsup _{n \rightarrow \infty} a_{n}^{2}$ equals sup $S$, where $S$ is the set of all subsequential limits of $\left\{a_{n}^{2}\right\}_{n \geq 1}$. This completes the proof.

Remark 15. We must note that Lemma 14 holds trivially if the sequence $\left\{a_{n}\right\}_{n \geq 1}$ was an unbounded sequence of nonnegative real numbers.

Remark 16. In the sequel, we shall make the following assumptions: $H$ is a real Hilbert space; $T^{\prime}: H \rightarrow H$ is a continuous pseudocontractive mapping; $T_{j}: H \rightarrow H, j=$ $1,2,3, \ldots$ is a countable infinite family of nonexpansive mappings; $f: H \times H \rightarrow \mathbb{R}$ is a bifunction satisfying conditions $(A 1)-(A 4) ; \Phi: H \rightarrow \mathbb{R} \cup\{+\infty\}$ a proper lower semicontinuous convex function; $\Theta: H \rightarrow H$ a continuous monotone mapping; $u \in H$ is a fixed vector; $A: H \rightarrow H$ is a strongly positive bounded linear operator with coefficient $\gamma ; B: H \rightarrow H$ is an $\eta$-inverse strongly monotone mapping. The sequences $\left\{r_{n}\right\}_{n \geq 1},\left\{\alpha_{n}\right\}_{n \geq 1},\left\{\beta_{n}\right\}_{n \geq 1}$, and $\left\{\lambda_{n}\right\}_{n \geq 1}$ are real sequences such that $r_{n}>0$ for all $n \in \mathbb{N}, \lim _{n \rightarrow \infty} r_{n}=r_{0}>0 ; 0<\alpha_{n}<1$ for all $n \in$ $\mathbb{N}, \lim _{n \rightarrow \infty} \alpha_{n}=0, \sum_{n=1}^{\infty} \alpha_{n}=+\infty ; 0<\beta_{n}<1$ for all $n \in \mathbb{N}, 0<\liminf _{n \rightarrow \infty} \beta_{n} \leq \limsup _{n \rightarrow \infty} \beta_{n}<1 ; 0<$ $\lambda_{n} \leq b<1$ for all $n \in \mathbb{N}$ and for some constant $b \in(0,1)$. $\xi$ is a real constant such that $0<\xi<2 \eta ; \Omega=\operatorname{Fix}\left(T^{\prime}\right) \cap$ $\operatorname{GMEP}(f, \Phi, \Theta) \cap B^{-1}(0) \cap \bigcap_{j=1}^{\infty} \operatorname{Fix}\left(T_{j}\right) \neq \varnothing$. For $r>0, F_{r}$ and $G_{r}$ are as in Lemmas 8 and 9, respectively.

We shall study the strong convergence of the sequence $\left\{x_{n}\right\}_{n \geq 1}$ generated iteratively from arbitrary $x_{1} \in H$ by

$$
\begin{aligned}
x_{n+1}= & \alpha_{n} u+\beta_{n} x_{n} \\
& +\left(\left(1-\beta_{n}\right) I-\alpha_{n} A\right) W_{n}(I-\xi B) F_{r_{n}} G_{r_{n}} x_{n}, \quad n \geq 1
\end{aligned}
$$

to a unique solution $x^{\prime} \in \Omega$ of the variational inequality

$$
\left\langle u-A x^{\prime}, x-x^{\prime}\right\rangle \leq 0 \quad \forall x \in \Omega .
$$

Lemma 17. Suppose that the conditions of Remark 16 are satisfied, then $\left\{x_{n}\right\}_{n \geq 1}$ defined by (74) is bounded.

Proof. Observe that for all $x, y \in H$,

$$
\begin{aligned}
\|(x- & \xi B x)-(y-\xi B y) \|^{2} \\
= & \|x-y-\xi(B x-B y)\|^{2} \\
= & \|x-y\|^{2}-2 \xi\langle B x-B y, x-y\rangle \\
& +\xi^{2}\|B x-B y\|^{2} \\
\leq & \|x-y\|^{2}-2 \xi \eta\|B x-B y\|^{2} \\
& +\xi^{2}\|B x-B y\|^{2}=\|x-y\|^{2} \\
& -\xi(2 \eta-\xi)\|B x-B y\|^{2} .
\end{aligned}
$$

Thus, since $0<\xi<2 \eta$, we get from (76) that

$$
\|(x-\xi B x)-(y-\xi B y)\| \leq\|x-y\| \quad \forall x, y \in H .
$$

It thus follows that for $p \in \Omega$,

$$
\begin{aligned}
& \left\|(I-\xi B) F_{r_{n}} G_{r_{n}} x_{n}-p\right\|^{2} \\
& =\left\|(I-\xi B) F_{r_{n}} G_{r_{n}} x_{n}-(I-\xi B) p\right\|^{2} \\
& \quad \leq\left\|F_{r_{n}} G_{r_{n}} x_{n}-p\right\|^{2} \leq\left\|x_{n}-p\right\|^{2} .
\end{aligned}
$$

Next, we may (without loss of generality) assume that $\alpha_{n}<$ $\|A\|^{-1}\left(1-\beta_{n}\right)$ for all $n \in \mathbb{N}$ since $\lim _{n \rightarrow \infty} \alpha_{n}=0$. So, for each $x \in H$ such that $\|x\|=1$, we have

$$
\begin{aligned}
\left\langle\left(\left(1-\beta_{n}\right) I-\alpha_{n} A\right) x, x\right\rangle \\
\quad=\left(1-\beta_{n}\right)-\alpha_{n}\langle A x, x\rangle \\
\quad \geq\left(1-\beta_{n}\right)-\alpha_{n}\|A\| \\
\quad>\left(1-\beta_{n}\right)-\|A\|^{-1}\left(1-\beta_{n}\right)\|A\|=0 .
\end{aligned}
$$

Thus, we have that for all $n \in \mathbb{N}$, the operator $\left(1-\beta_{n}\right) I-\alpha_{n} A$ is a positive bounded linear operator, so that

$$
\begin{aligned}
\|(1- & \left.\beta_{n}\right) I-\alpha_{n} A \| \\
& =\sup _{\|x\|=1}\left\langle\left(\left(1-\beta_{n}\right) I-\alpha_{n} A\right) x, x\right\rangle \\
& =\sup _{\|x\|=1}\left(1-\beta_{n}-\alpha_{n}\langle A x, x\rangle\right) \\
& \leq 1-\beta_{n}-\alpha_{n} \gamma .
\end{aligned}
$$


Therefore, for $p \in \Omega$, we obtain from (74) using (78) and (80) that

$$
\begin{aligned}
\left\|x_{n+1}-p\right\|= & \| \alpha_{n} u+\beta_{n} x_{n}+\left(\left(1-\beta_{n}\right) I-\alpha_{n} A\right) \\
& \times W_{n}(I-\xi B) F_{r_{n}} G_{r_{n}} x_{n}-p \| \\
= & \|\left(\left(1-\beta_{n}\right) I-\alpha_{n} A\right)\left(W_{n}(I-\xi B) F_{r_{n}} G_{r_{n}} x_{n}-p\right) \\
+ & \alpha_{n}(u-A p)+\beta_{n}\left(x_{n}-p\right) \| \\
\leq & \left(1-\beta_{n}-\alpha_{n} \gamma\right)\left\|W_{n}(I-\xi B) F_{r_{n}} G_{r_{n}} x_{n}-p\right\| \\
& +\beta_{n}\left\|x_{n}-p\right\|+\alpha_{n}\|u-A p\| \\
\leq & \left(1-\alpha_{n} \gamma\right)\left\|x_{n}-p\right\|+\alpha_{n}\|u-A p\| .
\end{aligned}
$$

It is thus easy to show by mathematical induction (using (81)) that

$$
\left\|x_{n}-p\right\| \leq \max \left\{\left\|x_{1}-p\right\|, \frac{1}{\gamma}\|u-A p\|\right\} .
$$

So, $\left\{x_{n}\right\}_{n \geq 1}$ is bounded. Consequently, $\left\{W_{n}(I-\xi B) F_{r_{n}} G_{r_{n}}\right.$. $\left.x_{n}\right\}_{n \geq 1},\left\{(I-\xi B) F_{r_{n}} G_{r_{n}} x_{n}\right\}_{n \geq 1},\left\{F_{r_{n}} G_{r_{n}} x_{n}\right\}_{n \geq 1}$ and $\left\{G_{r_{n}} x_{n}\right\}_{n \geq 1}$ are all bounded.

Lemma 18. Let $\left\{x_{n}\right\}_{n \geq 1}$ be given by (74). Suppose that conditions of Remark 16 are satisfied, then

$$
\lim _{n \rightarrow \infty}\left\|x_{n+1}-x_{n}\right\|=0
$$

Proof. Set $t_{n}:=\left(1 /\left(1-\beta_{n}\right)\right)\left(x_{n+1}-\beta_{n} x_{n}\right)$, then $x_{n+1}=\beta_{n} x_{n}+$ $\left(1-\beta_{n}\right) t_{n}$. Thus,

$$
\begin{aligned}
t_{n+1}-t_{n}= & \frac{1}{1-\beta_{n+1}}\left(x_{n+2}-\beta_{n+1} x_{n+1}\right) \\
& -\frac{1}{1-\beta_{n}}\left(x_{n+1}-\beta_{n} x_{n}\right) \\
= & \frac{1}{1-\beta_{n+1}}\left(\alpha_{n+1} u+\left[\left(1-\beta_{n+1}\right) I-\alpha_{n+1} A\right]\right. \\
& \quad \frac{1}{1-\beta_{n}}\left(\alpha_{n} u+\left[\left(1-\beta_{n}\right) I-\alpha_{n} A\right]\right. \\
& \left.\times W_{n}(I-\xi B) F_{r_{n}} G_{r_{n}} x_{n}\right)
\end{aligned}
$$

$$
\begin{aligned}
= & \frac{\alpha_{n+1}}{1-\beta_{n+1}}\left(u-A W_{n+1}(I-\xi B) F_{r_{n+1}} G_{r_{n+1}} x_{n+1}\right) \\
& -\frac{\alpha_{n}}{1-\beta_{n}}\left(u-A W_{n}(I-\xi B) F_{r_{n}} G_{r_{n}} x_{n}\right) \\
& +W_{n+1}(I-\xi B) F_{r_{n+1}} G_{r_{n+1}} x_{n+1} \\
& -W_{n}(I-\xi B) F_{r_{n}} G_{r_{n}} x_{n} \\
& +W_{n+1}(I-\xi B) F_{r_{n}} G_{r_{n}} x_{n} \\
& -W_{n+1}(I-\xi B) F_{r_{n}} G_{r_{n}} x_{n} .
\end{aligned}
$$

Thus, for $p \in \Omega$ (using (84), Lemmas 3, 13, and 12), we obtain (for some constant $M_{0}>0$ ) the following:

$$
\begin{aligned}
& \left\|t_{n+1}-t_{n}\right\|-\left\|x_{n+1}-x_{n}\right\| \\
& \begin{aligned}
\leq & {\left[\frac{\alpha_{n+1}}{1-\beta_{n+1}}+\frac{\alpha_{n}}{1-\beta_{n}}\right] M_{0} } \\
& +\left\|W_{n+1}(I-\xi B) F_{r_{n}} G_{r_{n}} x_{n}-W_{n}(I-\xi B) F_{r_{n}} G_{r_{n}} x_{n}\right\|
\end{aligned} \\
& \begin{aligned}
\leq & {\left[\frac{\alpha_{n+1}}{1-\beta_{n+1}}+\frac{\alpha_{n}}{1-\beta_{n}}\right] M_{0} } \\
& +\left\|W_{n+1}(I-\xi B) F_{r_{n}} G_{r_{n}} x_{n}-W_{n}(I-\xi B) F_{r_{n}} G_{r_{n}} x_{n}\right\|
\end{aligned} \\
& +\| W_{n+1}(I-\xi B) F_{r_{n+1}} G_{r_{n+1}} x_{n+1} \\
& -W_{n+1}(I-\xi B) F_{r_{n}} G_{r_{n}} x_{n}\|-\| x_{n+1}-x_{n} \| \\
& \leq\left[\frac{\alpha_{n+1}}{1-\beta_{n+1}}+\frac{\alpha_{n}}{1-\beta_{n}}\right] M_{0} \\
& +2 \prod_{j=1}^{n} \lambda_{j}\left\|(I-\xi B) F_{r_{n}} G_{r_{n}} x_{n}-p\right\| \\
& +\left\|F_{r_{n+1}} G_{r_{n+1}} x_{n+1}-F_{r_{n+1}} G_{r_{n}} x_{n}\right\| \\
& +\left\|F_{r_{n+1}} G_{r_{n}} x_{n}-F_{r_{n}} G_{r_{n}} x_{n}\right\|-\left\|x_{n+1}-x_{n}\right\| \\
& \leq\left[\frac{\alpha_{n+1}}{1-\beta_{n+1}}+\frac{\alpha_{n}}{1-\beta_{n}}\right] M_{0} \\
& +2 \prod_{j=1}^{n} \lambda_{j}\left\|F_{r_{n}} G_{r_{n}} x_{n}-p\right\|+\left\|G_{r_{n+1}} x_{n+1}-G_{r_{n}} x_{n}\right\| \\
& +\left\|F_{r_{n+1}} G_{r_{n}} x_{n}-F_{r_{n}} G_{r_{n}} x_{n}\right\|-\left\|x_{n+1}-x_{n}\right\| \\
& \leq\left[\frac{\alpha_{n+1}}{1-\beta_{n+1}}+\frac{\alpha_{n}}{1-\beta_{n}}\right] M_{0}+2 \prod_{j=1}^{n} \lambda_{j}\left\|F_{r_{n}} G_{r_{n}} x_{n}-p\right\| \\
& +\left\|G_{r_{n+1}} x_{n+1}-G_{r_{n+1}} x_{n}\right\|+\left\|G_{r_{n+1}} x_{n}-G_{r_{n}} x_{n}\right\| \\
& +\left\|F_{r_{n+1}} G_{r_{n}} x_{n}-F_{r_{n}} G_{r_{n}} x_{n}\right\|-\left\|x_{n+1}-x_{n}\right\| \\
& \leq\left[\frac{\alpha_{n+1}}{1-\beta_{n+1}}+\frac{\alpha_{n}}{1-\beta_{n}}\right] M_{0}
\end{aligned}
$$




$$
\begin{aligned}
& +2 \prod_{j=1}^{n} \lambda_{j}\left\|F_{r_{n}} G_{r_{n}} x_{n}-p\right\|+\frac{\left|r_{n+1}-r_{n}\right|}{r_{n}} \\
& \times\left[\left(\left\|F_{r_{n}} G_{r_{n}} x_{n}\right\|+2\left\|G_{r_{n}} x_{n}\right\|+\left\|x_{n}\right\|\right)\right] .
\end{aligned}
$$

$$
\begin{aligned}
\leq & \left\|x_{n+1}-x_{n}\right\|+\alpha_{n} M_{1} \\
& +\beta_{n}\left\|x_{n}-W_{n}(I-\xi B) F_{r_{n}} G_{r_{n}} x_{n}\right\| .
\end{aligned}
$$

Since $0<\lambda_{n}<b<1$ for all $n \in \mathbb{N}$ and for some constant $b \in(0,1)$, it is thus easy to see that $\lim _{n \rightarrow \infty} \prod_{j=1}^{n} \lambda_{j}=0$. So, we obtain from (85) that

$$
\limsup _{n \rightarrow \infty}\left(\left\|t_{n+1}-t_{n}\right\|-\left\|x_{n+1}-x_{n}\right\|\right) \leq 0 .
$$

Lemma 6 therefore gives

$$
\lim _{n \rightarrow \infty}\left\|t_{n}-x_{n}\right\|=0
$$

but

$$
x_{n+1}-x_{n}=\left(1-\beta_{n}\right)\left(t_{n}-x_{n}\right) \text {. }
$$

Hence,

$$
\lim _{n \rightarrow \infty}\left\|x_{n+1}-x_{n}\right\|=0
$$

Lemma 19. Let $\left\{x_{n}\right\}_{n>1}$ be given by (74). Suppose conditions of Remark 16 are satisfied, then

$$
\begin{aligned}
\lim _{n \rightarrow \infty} & \left\|W_{n}(I-\xi B) F_{r_{n}} G_{r_{n}} x_{n}-x_{n}\right\| \\
& =\lim _{n \rightarrow \infty}\left\|x_{n}-F_{r_{n}} G_{r_{n}} x_{n}\right\| \\
& =\lim _{n \rightarrow \infty}\left\|B F_{r_{n}} G_{r_{n}} x_{n}\right\| \\
& =\lim _{n \rightarrow \infty}\left\|W_{n}(I-\xi B) F_{r_{n}} G_{r_{n}} x_{n}-F_{r_{n}} G_{r_{n}} x_{n}\right\| \\
& =\lim _{n \rightarrow \infty}\left\|x_{n}-G_{r_{n}} x_{n}\right\|=0 .
\end{aligned}
$$

Proof. Observe that for some constant $M_{1}>0$,

$$
\begin{aligned}
& \left\|x_{n}-W_{n}(I-\xi B) F_{r_{n}} G_{r_{n}} x_{n}\right\| \\
& \leq\left\|x_{n}-x_{n+1}\right\|+\left\|x_{n+1}-W_{n}(I-\xi B) F_{r_{n}} G_{r_{n}} x_{n}\right\| \\
& =\left\|x_{n}-x_{n+1}\right\| \\
& \quad+\| \alpha_{n} u+\beta_{n} x_{n}+\left[\left(1-\beta_{n}\right) I-\alpha_{n} A\right] \\
& \quad \times W_{n}(I-\xi B) F_{r_{n}} G_{r_{n}} x_{n} \\
& \quad-W_{n}(I-\xi B) F_{r_{n}} G_{r_{n}} x_{n} \| \\
& \leq\left\|x_{n+1}-x_{n}\right\| \\
& +\alpha_{n}\left\|u-A W_{n}(I-\xi B) F_{r_{n}} G_{r_{n}} x_{n}\right\| \\
& \quad+\beta_{n}\left\|x_{n}-W_{n}(I-\xi B) F_{r_{n}} G_{r_{n}} x_{n}\right\|
\end{aligned}
$$

So, we obtain from (91) that

$$
\begin{aligned}
& \left\|W_{n}(I-\xi B) F_{r_{n}} G_{r_{n}} x_{n}-x_{n}\right\| \\
& \quad \leq \frac{1}{1-\beta_{n}}\left(\left\|x_{n}-x_{n+1}\right\|+\alpha_{n} M_{1}\right) .
\end{aligned}
$$

Using (89) and the fact that $\lim _{n \rightarrow \infty} \alpha_{n}=0$, we obtain from (92) that

$$
\lim _{n \rightarrow \infty}\left\|W_{n}(I-\xi B) F_{r_{n}} G_{r_{n}} x_{n}-x_{n}\right\|=0 .
$$

Next, observe that for fixed $p \in \Omega$ and using (2) of Lemma 9, we obtain the following:

$$
\begin{gathered}
\left\|G_{r_{n}} x_{n}-p\right\|^{2} \leq\left\langle G_{r_{n}} x_{n}-p, x_{n}-p\right\rangle \\
=\frac{1}{2}\left[\left\|G_{r_{n}} x_{n}-p\right\|^{2}+\left\|x_{n}-p\right\|^{2}\right. \\
\left.\quad-\left\|x_{n}-G_{r_{n}} x_{n}\right\|^{2}\right],
\end{gathered}
$$

so that

$$
\begin{aligned}
\left\|G_{r_{n}} x_{n}-p\right\|^{2} \leq & \left\|x_{n}-p\right\|^{2} \\
& -\left\|x_{n}-G_{r_{n}} x_{n}\right\|^{2} .
\end{aligned}
$$

Furthermore, using (2) of Lemma 8, we obtain

$$
\begin{gathered}
\left\|F_{r_{n}} G_{r_{n}} x_{n}-p\right\|^{2} \\
\leq\left\langle F_{r_{n}} G_{r_{n}} x_{n}-p, G_{r_{n}} x_{n}-p\right\rangle \\
=\frac{1}{2}\left[\left\|F_{r_{n}} G_{r_{n}} x_{n}-p\right\|^{2}+\left\|G_{r_{n}} x_{n}-p\right\|^{2}\right. \\
\left.-\left\|G_{r_{n}} x_{n}-F_{r_{n}} G_{r_{n}} x_{n}\right\|^{2}\right],
\end{gathered}
$$

so that

$$
\begin{aligned}
\left\|F_{r_{n}} G_{r_{n}} x_{n}-p\right\|^{2} \leq & \left\|G_{r_{n}} x_{n}-p\right\|^{2} \\
& -\left\|G_{r_{n}} x_{n}-F_{r_{n}} G_{r_{n}} x_{n}\right\|^{2} .
\end{aligned}
$$


Moreover, using the recursion formula (74), Lemma 4, and convexity of $\|\cdot\|^{2}$, we have

$$
\begin{aligned}
\left\|x_{n+1}-p\right\|^{2}= & \| \alpha_{n} u+\beta_{n} x_{n}+\left[\left(1-\beta_{n}\right) I-\alpha_{n} A\right] \\
& \times W_{n}(I-\xi B) F_{r_{n}} G_{r_{n}} x_{n}-p \|^{2} \\
\leq & \beta_{n}\left\|x_{n}-p\right\|^{2}+\left(1-\beta_{n}\right) \\
& \times\left\|W_{n}(I-\xi B) F_{r_{n}} G_{r_{n}} x_{n}-p\right\|^{2} \\
& +2 \alpha_{n}\left\langle u-A W_{n}(I-\xi B)\right. \\
& \left.\times F_{r_{n}} G_{r_{n}} x_{n}, x_{n+1}-p\right\rangle \\
\leq & \beta_{n}\left\|x_{n+1}-p\right\|^{2} \\
& +2 \beta_{n}\left\|x_{n+1}-p\right\|\left\|x_{n}-x_{n+1}\right\| \\
& +\beta_{n}\left\|x_{n+1}-x_{n}\right\|^{2} \\
& \times\left(1-\beta_{n}\right)\left\|(I-\xi B) F_{r_{n}} G_{r_{n}} x_{n}-p\right\|^{2} \\
& +2 \alpha_{n}\left\langle u-A W_{n}(I-\xi B)\right. \\
& \left.\times F_{r_{n}} G_{r_{n}} x_{n}, x_{n+1}-p\right\rangle .
\end{aligned}
$$

Thus, using (76) and (97), we obtain from (98) the following:

$$
\begin{aligned}
\| x_{n+1} & -p \|^{2} \\
\leq & \left\|(I-\xi B) F_{r_{n}} G_{r_{n}} x_{n}-p\right\|^{2} \\
& +\frac{\beta_{n}}{1-\beta_{n}}\left(2\left\|x_{n+1}-p\right\|+\left\|x_{n+1}-x_{n}\right\|\right) \\
& \times\left\|x_{n+1}-x_{n}\right\|+\frac{2 \alpha_{n}}{1-\beta_{n}} \\
& \times\left\langle u-A W_{n}(I-\xi B) F_{r_{n}} G_{r_{n}} x_{n}, x_{n+1}-p\right\rangle \\
\leq & \left\|F_{r_{n}} G_{r_{n}} x_{n}-p\right\|^{2}-\xi(2 \eta-\xi)\left\|B F_{r_{n}} G_{r_{n}} x_{n}\right\|^{2} \\
& +\frac{\beta_{n}}{1-\beta_{n}}\left(2\left\|x_{n+1}-p\right\|+\left\|x_{n+1}-x_{n}\right\|\right) \\
& \times\left\|x_{n+1}-x_{n}\right\|+\frac{2 \alpha_{n}}{1-\beta_{n}} \\
\leq & \left\|x_{n}-p\right\|^{2}-\left\|G_{r_{n}} x_{n}-F_{r_{n}} G_{r_{n}} x_{n}\right\|^{2} \\
& -\xi(2 \eta-\xi)\left\|B F_{r_{n}} G_{r_{n}} x_{n}\right\|^{2} \\
& \times\left\langle u-A W_{n}(I-\xi B) F_{r_{n}} G_{r_{n}} x_{n}, x_{n+1}-p\right\rangle
\end{aligned}
$$

$$
\begin{aligned}
& +\frac{\beta_{n}}{1-\beta_{n}}\left(2\left\|x_{n+1}-p\right\|+\left\|x_{n+1}-x_{n}\right\|\right) \\
& \times\left\|x_{n+1}-x_{n}\right\|+\frac{2 \alpha_{n}}{1-\beta_{n}} \\
& \times\left\langle u-A W_{n}(I-\xi B) F_{r_{n}} G_{r_{n}} x_{n}, x_{n+1}-p\right\rangle .
\end{aligned}
$$

So, from (99), we have (for some real constant $M_{2}>0$ )

$$
\begin{aligned}
\| x_{n}- & F_{r_{n}} G_{r_{n}} x_{n}\left\|^{2}+\xi(2 \eta-\xi)\right\| B F_{r_{n}} G_{r_{n}} x_{n} \|^{2} \\
\leq & \left\|x_{n}-p\right\|^{2}-\left\|x_{n+1}-p\right\|^{2}+\frac{\beta_{n}}{1-\beta_{n}} \\
& \times\left(2\left\|x_{n+1}-p\right\|+\left\|x_{n+1}-x_{n}\right\|\right) \\
& \times\left\|x_{n+1}-x_{n}\right\|+\frac{2 \alpha_{n}}{1-\beta_{n}} \\
& \times\left\langle u-A W_{n}(I-\xi B) F_{r_{n}} G_{r_{n}} x_{n}, x_{n+1}-p\right\rangle \\
\leq & \left(\alpha_{n}+\left\|x_{n+1}-x_{n}\right\|\right) M_{2} .
\end{aligned}
$$

From (100), we have

$$
\begin{aligned}
\lim _{n \rightarrow \infty}( & \left\|x_{n}-F_{r_{n}} G_{r_{n}} x_{n}\right\|^{2} \\
& \left.+\xi(2 \eta-\xi)\left\|B F_{r_{n}} G_{r_{n}} x_{n}\right\|^{2}\right)=0,
\end{aligned}
$$

and since $\xi(2 \eta-\xi)>0$, then using (101) and applying sandwich theorem, we obtain

$$
\begin{gathered}
\lim _{n \rightarrow \infty}\left\|x_{n}-F_{r_{n}} G_{r_{n}} x_{n}\right\|=0, \\
\lim _{n \rightarrow \infty}\left\|B F_{r_{n}} G_{r_{n}} x_{n}\right\|=0 .
\end{gathered}
$$

Furthermore, observe that

$$
\begin{aligned}
& \left\|W_{n}(I-\xi B) F_{r_{n}} G_{r_{n}} x_{n}-F_{r_{n}} G_{r_{n}} x_{n}\right\| \\
& \quad \leq\left\|W_{n}(I-\xi B) F_{r_{n}} G_{r_{n}} x_{n}-x_{n}\right\|+\left\|x_{n}-F_{r_{n}} G_{r_{n}} x_{n}\right\| .
\end{aligned}
$$

Hence, using (93), (102), (103), and (104), we have

$$
\lim _{n \rightarrow \infty}\left\|W_{n}(I-\xi B) F_{r_{n}} G_{r_{n}} x_{n}-F_{r_{n}} G_{r_{n}} x_{n}\right\|=0 .
$$


Besides, we obtain from the second line of inequality (99) (using (95) and the fact that $F_{r_{n}}$ is firmly nonexpansive; thus nonexpansive) that for some constant $M^{\prime}>0$,

$$
\begin{aligned}
&\left\|x_{n+1}-p\right\|^{2} \\
& \leq\left\|F_{r_{n}} G_{r_{n}} x_{n}-p\right\|^{2} \\
&-\xi(2 \eta-\xi)\left\|B F_{r_{n}} G_{r_{n}} x_{n}\right\|^{2} \\
&+\frac{\beta_{n}}{1-\beta_{n}}\left(2\left\|x_{n+1}-p\right\|+\left\|x_{n+1}-x_{n}\right\|\right) \\
& \times\left\|x_{n+1}-x_{n}\right\|+\frac{2 \alpha_{n}}{1-\beta_{n}} \\
& \times\left\langle u-A W_{n}(I-\xi B) F_{r_{n}} G_{r_{n}} x_{n}, x_{n+1}-p\right\rangle \\
& \leq\left\|G_{r_{n}} x_{n}-p\right\|^{2}+\left[\left\|x_{n+1}-x_{n}\right\|+\alpha_{n}\right] M^{\prime} \\
& \leq\left\|x_{n}-p\right\|^{2} \\
&-\left\|x_{n}-G_{r_{n}} x_{n}\right\|^{2}+\left[\left\|x_{n+1}-x_{n}\right\|+\alpha_{n}\right] M^{\prime} .
\end{aligned}
$$

So, we obtain from (106) that for some constant $M^{\prime \prime}>0$,

$$
\begin{aligned}
& \left\|x_{n}-G_{r_{n}} x_{n}\right\| \\
& \leq\left\|x_{n}-p\right\|^{2} \\
& \quad-\left\|x_{n+1}-p\right\|^{2}+\left[\left\|x_{n+1}-x_{n}\right\|+\alpha_{n}\right] M^{\prime} \\
& \quad \leq\left\|x_{n+1}-x_{n}\right\| M^{\prime \prime}+\left[\left\|x_{n+1}-x_{n}\right\|+\alpha_{n}\right] M^{\prime} .
\end{aligned}
$$

Hence, $\lim _{n \rightarrow \infty}\left\|x_{n}-G_{r_{n}} x_{n}\right\|=0$.

Lemma 20. Let $\left\{x_{n}\right\}_{n \geq 1}$ be given by (74). Suppose that conditions of Remark 16 hold, then

$$
\lim _{n \rightarrow \infty}\left\|W_{n}(I-\xi B) F_{r_{n}} G_{r_{n}} x_{n}-(I-\xi B) F_{r_{n}} G_{r_{n}} x_{n}\right\|=0 .
$$

Proof. Let $p \in \Omega$, then we obtain

$$
\begin{aligned}
\|(I & -\xi B) F_{r_{n}} G_{r_{n}} x_{n}-p \|^{2} \\
& =\left\|F_{r_{n}} G_{r_{n}} x_{n}-\xi B F_{r_{n}} G_{r_{n}} x_{n}-p\right\|^{2} \\
& =\left\|\left(F_{r_{n}} G_{r_{n}} x_{n}-\xi B F_{r_{n}} G_{r_{n}} x_{n}\right)-(p-\xi B p)\right\|^{2}
\end{aligned}
$$

$$
\begin{aligned}
& =\frac{1}{2}\left[\left\|\left(F_{r_{n}} G_{r_{n}} x_{n}-\xi B F_{r_{n}} G_{r_{n}} x_{n}\right)-(p-\xi B p)\right\|^{2}\right. \\
& +\left\|(I-\xi B) F_{r_{n}} G_{r_{n}} x_{n}-p\right\|^{2} \\
& -\|\left(\left(F_{r_{n}} G_{r_{n}} x_{n}-\xi B F_{r_{n}} G_{r_{n}} x_{n}\right)-(p-\xi B p)\right) \\
& \left.\quad-\left((I-\xi B) F_{r_{n}} G_{r_{n}} x_{n}-p\right) \|^{2}\right] \\
& \leq \frac{1}{2}\left[\left\|F_{r_{n}} G_{r_{n}} x_{n}-p\right\|^{2}\right. \\
& +\left\|(I-\xi B) F_{r_{n}} G_{r_{n}} x_{n}-p\right\|^{2} \\
& \quad-\left\|\left(F_{r_{n}} G_{r_{n}} x_{n}-p\right)-\left((I-\xi B) F_{r_{n}} G_{r_{n}} x_{n}-p\right)\right\|^{2} \\
& +2 \xi\left\langle F_{r_{n}} G_{r_{n}} x_{n}-(I-\xi B)\right. \\
& \left.\quad \times F_{r_{n}} G_{r_{n}} x_{n}, B F_{r_{n}} G_{r_{n}} x_{n}\right\rangle \\
& \left.\quad-\xi^{2}\left\|B F_{r_{n}} G_{r_{n}} x_{n}\right\|^{2}\right] .
\end{aligned}
$$

This inequality implies the following:

$$
\begin{aligned}
\|(I- & \xi B) F_{r_{n}} G_{r_{n}} x_{n}-p \|^{2} \\
\leq & \left\|x_{n}-p\right\|^{2}-\left\|F_{r_{n}} G_{r_{n}} x_{n}-(I-\xi B) F_{r_{n}} G_{r_{n}} x_{n}\right\|^{2} \\
& +2\left\|F_{r_{n}} G_{r_{n}} x_{n}-(I-\xi B) F_{r_{n}} G_{r_{n}} x_{n}\right\| \\
& \times\left\|B F_{r_{n}} G_{r_{n}} x_{n}\right\|-\xi^{2}\left\|B F_{r_{n}} G_{r_{n}} x_{n}\right\|^{2} \\
\leq & \left\|x_{n}-x_{n+1}\right\|^{2}+2\left\|x_{n+1}-p\right\| \\
& \times\left\|x_{n+1}-x_{n}\right\|+\left\|x_{n+1}-p\right\|^{2} \\
& -\left\|F_{r_{n}} G_{r_{n}} x_{n}-(I-\xi B) F_{r_{n}} G_{r_{n}} x_{n}\right\|^{2} \\
& +2\left\|F_{r_{n}} G_{r_{n}} x_{n}-(I-\xi B) F_{r_{n}} G_{r_{n}} x_{n}\right\| \\
& \times\left\|B F_{r_{n}} G_{r_{n}} x_{n}\right\| .
\end{aligned}
$$

But from the first line of inequality (99), we have

$$
\begin{aligned}
\| x_{n+1} & -p \|^{2} \\
\leq & \left\|(I-\xi B) F_{r_{n}} G_{r_{n}} x_{n}-p\right\|^{2} \\
& +\frac{\beta_{n}}{1-\beta_{n}}\left(2\left\|x_{n+1}-p\right\|+\left\|x_{n+1}-x_{n}\right\|\right) \\
& \times\left\|x_{n+1}-x_{n}\right\|+\frac{2 \alpha_{n}}{1-\beta_{n}} \\
& \times\left\langle u-A W_{n}(I-\xi B) F_{r_{n}} G_{r_{n}} x_{n}, x_{n+1}-p\right\rangle .
\end{aligned}
$$


So, for some real constant $M_{2}>0$, we obtain from (111) that

$$
\begin{aligned}
\left\|x_{n+1}-p\right\|^{2} \leq & \left\|(I-\xi B) F_{r_{n}} G_{r_{n}} x_{n}-p\right\|^{2} \\
& +\left(\alpha_{n}+\left\|x_{n+1}-x_{n}\right\|\right) M_{2} .
\end{aligned}
$$

Using (112) in (110) and rearranging terms, we obtain (for some real constant $M_{3}>0$ ) that

$$
\begin{aligned}
& \left\|F_{r_{n}} G_{r_{n}} x_{n}-(I-\xi B) F_{r_{n}} G_{r_{n}} x_{n}\right\|^{2} \\
& \quad \leq\left(\alpha_{n}+\left\|x_{n+1}-x_{n}\right\|+\left\|B F_{r_{n}} G_{r_{n}} x_{n}\right\|\right) M_{3} .
\end{aligned}
$$

So, (113) implies that

$$
\lim _{n \rightarrow \infty}\left\|F_{r_{n}} G_{r_{n}} x_{n}-(I-\xi B) F_{r_{n}} G_{r_{n}} x_{n}\right\|=0 .
$$

Hence,

$$
\begin{aligned}
& \left\|(I-\xi B) F_{r_{n}} G_{r_{n}} x_{n}-x_{n}\right\| \\
& \leq\left\|(I-\xi B) F_{r_{n}} G_{r_{n}} x_{n}-F_{r_{n}} G_{r_{n}} x_{n}\right\| \\
& \quad+\left\|F_{r_{n}} G_{r_{n}} x_{n}-x_{n}\right\| \longrightarrow \text { as } n \longrightarrow \infty, \\
& \left\|W_{n}(I-\xi B) F_{r_{n}} G_{r_{n}} x_{n}-(I-\xi B) F_{r_{n}} G_{r_{n}} x_{n}\right\| \\
& \leq\left\|W_{n}(I-\xi B) F_{r_{n}} G_{r_{n}} x_{n}-x_{n}\right\| \\
& \quad+\left\|x_{n}-(I-\xi B) F_{r_{n}} G_{r_{n}} x_{n}\right\| .
\end{aligned}
$$

The last inequality implies that

$$
\lim _{n \rightarrow \infty}\left\|W_{n}(I-\xi B) F_{r_{n}} G_{r_{n}} x_{n}-(I-\xi B) F_{r_{n}} G_{r_{n}} x_{n}\right\|=0 .
$$

Lemma 21. Let $\left\{x_{n}\right\}_{n \geq 1}$ be given by (74) and let $x \in \Omega$ be the unique solution of the variational inequality (75). Suppose that conditions of Remark 16 hold, then

$$
\limsup _{n \rightarrow \infty}\left\langle u-A x^{\prime}, x_{n}-x^{\prime}\right\rangle \leq 0 .
$$

Proof. Let $\left\{x_{n_{j}}\right\}_{j \geq 1}$ be a subsequence of $\left\{x_{n}\right\}_{n \geq 1}$ such that

$$
\begin{aligned}
& \limsup _{n \rightarrow \infty}\left\langle u-A x^{\prime}, x_{n}-x^{\prime}\right\rangle \\
& =\lim _{j \rightarrow \infty}\left\langle u-A x^{\prime}, x_{n_{j}}-x^{\prime}\right\rangle .
\end{aligned}
$$

Since every real Hilbert space $H$ is a uniformly convex real Banach space and $\left\{x_{n}\right\}_{n \geq 1}$ is a bounded sequence in $H$, then we obtain by Lemma 11 that there exists a unique $x^{*} \in H$ such that $x^{*}$ is the asymptotic center of the sequence $\left\{x_{n_{j}}\right\}_{j \geq 1}$. We first show that $x^{*} \in \Omega$. Recall that $r_{0}=\lim _{n \rightarrow \infty} r_{n}$ and that by Lemma $1, W x:=\lim _{n \rightarrow \infty} W_{n} x$ exists for all $x \in H$ and by Lemma 2 that $\operatorname{Fix}(W)=\bigcap_{n=1}^{\infty} \operatorname{Fix}\left(W_{n}\right)=$ $\bigcap_{j=1}^{\infty} \operatorname{Fix}\left(T_{j}\right)$; thus,

$$
\begin{aligned}
& \left\|x_{n_{j}}-W x^{*}\right\| \\
& \leq\left\|x_{n_{j}+1}-x_{n_{j}}\right\| \\
& +\| \alpha_{n_{j}} u+\beta_{n_{j}} x_{n_{j}}+\left[\left(1-\beta_{n_{j}}\right) I-\alpha_{n_{j}} A\right] \\
& \quad \times W_{n_{j}}(I-\xi B) F_{r_{n_{j}}} G_{r_{n_{j}}} x_{n_{j}}-W x^{*} \| \\
& \leq\left\|x_{n_{j}+1}-x_{n_{j}}\right\|+\beta_{n_{j}}\left\|x_{n_{j}}-W x^{*}\right\|+\left(1-\beta_{n_{j}}\right) \\
& \quad \times\left(\left\|W_{n_{j}}(I-\xi B) F_{r_{n_{j}}} G_{r_{n_{j}}} x_{n_{j}}-W_{n_{j}} x_{n_{j}}\right\|\right. \\
& \left.+\left\|W_{n_{j}} x_{n_{j}}-W_{n_{j}} x^{*}\right\|+\left\|W_{n_{j}} x^{*}-W x^{*}\right\|\right) \\
& +\alpha_{n_{j}}\left\|u-A W_{n_{j}}(I-\xi B) F_{r_{n_{j}}} G_{r_{n_{j}}} x_{n_{j}}\right\| \\
& \leq\left\|x_{n_{j}+1}-x_{n_{j}}\right\|+\beta_{n_{j}}\left\|x_{n_{j}}-W x^{*}\right\| \\
& +\left(1-\beta_{n_{j}}\right)\left(\left\|(I-\xi B) F_{r_{n_{j}}} G_{r_{n_{j}}} x_{n_{j}}-x_{n_{j}}\right\|\right. \\
& +\alpha_{n_{j}}\left\|u-A W_{n_{j}}(I-\xi B) F_{r_{n_{j}}} G_{r_{n_{j}}} x_{n_{j}}\right\| .
\end{aligned}
$$

Thus, we obtain from (119) that

$$
\begin{aligned}
\left\|x_{n_{j}}-W x^{*}\right\| & \\
\leq & \frac{1}{1-\beta_{n_{j}}}\left\|x_{n_{j}+1}-x_{n_{j}}\right\| \\
& +\left\|(I-\xi B) F_{r_{n_{j}}} G_{r_{n_{j}}} x_{n_{j}}-x_{n_{j}}\right\| \\
& +\left\|x_{n_{j}}-x^{*}\right\|+\left\|W_{n_{j}} x^{*}-W x^{*}\right\| \\
& +\frac{\alpha_{n_{j}}}{1-\beta_{n_{j}}}\left\|\mathcal{u}-A W_{n_{j}}(I-\xi B) F_{r_{n_{j}}} G_{r_{n_{j}}} x_{n_{j}}\right\|,
\end{aligned}
$$

so that

$$
\limsup _{j \rightarrow \infty}\left\|x_{n_{j}}-W x^{*}\right\| \leq \limsup _{j \rightarrow \infty}\left\|x_{n_{j}}-x^{*}\right\| .
$$

This implies by Definition 10 (with $C=H$ ) and uniqueness of $x^{*}$ that $W x^{*}=x^{*}$; that is, $x^{*} \in \bigcap_{j=1}^{\infty} \operatorname{Fix}\left(T_{j}\right)$. 
International Journal of Analysis

13

Next,

$$
\begin{aligned}
& \left\|x_{n_{j}}-F_{r_{0}} x^{*}\right\| \\
& \leq\left\|x_{n_{j}+1}-x_{n_{j}}\right\| \\
& +\| \alpha_{n_{j}} u+\beta_{n_{j}} x_{n_{j}}+\left[\left(1-\beta_{n_{j}}\right) I-\alpha_{n_{j}} A\right] \\
& \times W_{n_{j}}(I-\xi B) F_{r_{n_{j}}} G_{r_{n_{j}}} x_{n_{j}}-F_{r_{0}} x^{*} \| \\
& \leq\left\|x_{n_{j}+1}-x_{n_{j}}\right\|+\beta_{n_{j}}\left\|x_{n_{j}}-F_{r_{0}} x^{*}\right\|+\left(1-\beta_{n_{j}}\right) \\
& \times\left(\left\|W_{n_{j}}(I-\xi B) F_{r_{n_{j}}} G_{r_{n_{j}}} x_{n_{j}}-F_{r_{n_{j}}} G_{r_{n_{j}}} x_{n_{j}}\right\|\right. \\
& +\left\|F_{r_{n_{j}}} G_{r_{n_{j}}} x_{n_{j}}-F_{r_{n_{j}}} x_{n_{j}}\right\| \\
& \left.+\left\|F_{r_{n_{j}}} x_{n_{j}}-F_{r_{n_{j}}} x^{*}\right\|+\left\|F_{r_{n_{j}}} x^{*}-F_{r_{0}} x^{*}\right\|\right) \\
& +\alpha_{n_{j}}\left\|u-A W_{n_{j}}(I-\xi B) F_{r_{n_{j}}} G_{r_{n_{j}}} x_{n_{j}}\right\| \\
& \leq\left\|x_{n_{j}+1}-x_{n_{j}}\right\|+\beta_{n_{j}}\left\|x_{n_{j}}-F_{r_{0}} x^{*}\right\|+\left(1-\beta_{n_{j}}\right) \\
& \times\left(\left\|W_{n_{j}}(I-\xi B) F_{r_{n_{j}}} G_{r_{n_{j}}} x_{n_{j}}-F_{r_{n_{j}}} G_{r_{n_{j}}} x_{n_{j}}\right\|\right. \\
& +\left\|G_{r_{n_{j}}} x_{n_{j}}-x_{n_{j}}\right\|+\left\|x_{n_{j}}-x^{*}\right\| \\
& \left.+\frac{\left|r_{n_{j}}-r_{0}\right|}{r_{0}}\left[\left\|F_{r_{0}} x^{*}\right\|+\left\|x^{*}\right\|\right]\right) \\
& +\alpha_{n_{j}}\left\|u-A W_{n_{j}}(I-\xi B) F_{r_{n_{j}}} G_{r_{n_{j}}} x_{n_{j}}\right\|,
\end{aligned}
$$

so that from (122), we obtain

$$
\begin{aligned}
\left\|x_{n_{j}}-F_{r_{0}} x^{*}\right\| & \\
\leq & \frac{1}{1-\beta_{n_{j}}}\left\|x_{n_{j}+1}-x_{n_{j}}\right\| \\
& +\left\|W_{n_{j}}(I-\xi B) F_{r_{n_{j}}} G_{r_{n_{j}}} x_{n_{j}}-F_{r_{n_{j}}} G_{r_{n_{j}}} x_{n_{j}}\right\| \\
& +\left\|G_{r_{n_{j}}} x_{n_{j}}-x_{n_{j}}\right\|+\left\|x_{n_{j}}-x^{*}\right\| \\
& +\frac{\left|r_{n_{j}}-r_{0}\right|}{r_{0}}\left[\left\|F_{r_{0}} x^{*}\right\|+\left\|x^{*}\right\|\right] \\
& \times \frac{\alpha_{n_{j}}}{1-\beta_{n_{j}}}\left\|u-A W_{n_{j}}(I-\xi B) F_{r_{n_{j}}} G_{r_{n_{j}}} x_{n_{j}}\right\| .
\end{aligned}
$$

Thus,

$$
\limsup _{n \rightarrow \infty}\left\|x_{n_{j}}-F_{r_{0}} x^{*}\right\| \leq \limsup _{n \rightarrow \infty}\left\|x_{n_{j}}-x^{*}\right\|
$$

So, by Definition 10, we obtain that $F_{r_{0}} x^{*}=x^{*} \Leftrightarrow T^{\prime} x^{*}=$ $x^{*}$, that is, $x^{*} \in \operatorname{Fix}\left(T^{\prime}\right)$. Moreover,

$$
\begin{aligned}
& \left\|x_{n_{j}}-G_{r_{0}} x^{*}\right\| \\
& \leq\left\|x_{n_{j}+1}-x_{n_{j}}\right\| \\
& +\| \alpha_{n_{j}} u+\beta_{n_{j}} x_{n_{j}}+\left[\left(1-\beta_{n_{j}}\right) I-\alpha_{n_{j}} A\right] \\
& \times W_{n_{j}}(I-\xi B) F_{r_{n_{j}}} G_{r_{n_{j}}} x_{n_{j}}-G_{r_{0}} x^{*} \| \\
& \leq\left\|x_{n_{j}+1}-x_{n_{j}}\right\|+\beta_{n_{j}}\left\|x_{n_{j}}-G_{r_{0}} x^{*}\right\|+\left(1-\beta_{n_{j}}\right) \\
& \times\left(\left\|W_{n_{j}}(I-\xi B) F_{r_{n_{j}}} G_{r_{n_{j}}} x_{n_{j}}-F_{r_{n_{j}}} G_{r_{n_{j}}} x_{n_{j}}\right\|\right. \\
& +\left\|F_{r_{n_{j}}} G_{r_{n_{j}}} x_{n_{j}}-x_{n_{j}}\right\| \\
& +\left\|x_{n_{j}}-G_{r_{n_{j}}} x_{n_{j}}\right\|+\| G_{r_{n_{j}}} x_{n_{j}}-G_{r_{n_{j}}} x^{* \|} \\
& \left.+\left\|G_{r_{n_{j}}} x^{*}-G_{r_{0}} x^{*}\right\|\right) \\
& +\alpha_{n_{j}}\left\|u-A W_{n_{j}}(I-\xi B) F_{r_{n_{j}}} G_{r_{n_{j}}} x_{n_{j}}\right\| \\
& \leq\left\|x_{n_{j}+1}-x_{n_{j}}\right\|+\beta_{n_{j}}\left\|x_{n_{j}}-G_{r_{0}} x^{*}\right\|+\left(1-\beta_{n_{j}}\right) \\
& \times\left(\left\|W_{n_{j}}(I-\xi B) F_{r_{n_{j}}} G_{r_{n_{j}}} x_{n_{j}}-F_{r_{n_{j}}} G_{r_{n_{j}}} x_{n_{j}}\right\|\right. \\
& +\left\|F_{r_{n_{j}}} G_{r_{n_{j}}} x_{n_{j}}-x_{n_{j}}\right\| \\
& +\left\|x_{n_{j}}-G_{r_{n_{j}}} x_{n_{j}}\right\|+\left\|x_{n_{j}}-x^{*}\right\| \\
& \left.+\frac{\left|r_{n_{j}}-r_{0}\right|}{r_{0}}\left(\left\|G_{r_{0}} x^{*}\right\|+\left\|x^{*}\right\|\right)\right) \\
& +\alpha_{n_{j}}\left\|u-A W_{n_{j}}(I-\xi B) F_{r_{n_{j}}} G_{r_{n_{j}}} x_{n_{j}}\right\| .
\end{aligned}
$$


14

International Journal of Analysis

Thus, we obtain from (125) that

$$
\begin{aligned}
\left\|x_{n_{j}}-G_{r_{0}} x^{*}\right\| \leq \frac{1}{1-\beta_{n_{j}}}\left\|x_{n_{j}+1}-x_{n_{j}}\right\| \\
+\left\|W_{n_{j}}(I-\xi B) F_{r_{n_{j}}} G_{r_{n_{j}}} x_{n_{j}}-F_{r_{n_{j}}} G_{r_{n_{j}}} x_{n_{j}}\right\| \\
+\left\|F_{r_{n_{j}}} G_{r_{n_{j}}} x_{n_{j}}-x_{n_{j}}\right\|+\left\|x_{n_{j}}-G_{r_{n_{j}}} x_{n_{j}}\right\| \\
+\left\|x_{n_{j}}-x^{*}\right\|+\frac{\left|r_{n_{j}}-r_{0}\right|}{r_{0}}\left(\left\|G_{r_{0}} x^{*}\right\|+\left\|x^{*}\right\|\right) \\
+\frac{\alpha_{n_{j}}}{1-\beta_{n_{j}}}\left\|u-A W_{n_{j}}(I-\xi B) F_{r_{n_{j}}} G_{r_{n_{j}}} x_{n_{j}}\right\| .
\end{aligned}
$$

Thus,

$$
\limsup _{j \rightarrow \infty}\left\|x_{n_{j}}-G_{r_{0}} x^{*}\right\| \leq \underset{j \rightarrow \infty}{\limsup }\left\|x_{n_{j}}-x^{*}\right\|
$$

Definition 10 again implies that $G_{r_{0}} x^{*}=x^{*}$. Hence $x^{*} \epsilon$ $\operatorname{GMEP}(f, \Phi, \Theta)$.

Furthermore, since we have shown that $G_{r_{0}} x^{*}=x^{*}=$ $F_{r_{0}} x^{*}$, then making use of this fact and Lemma 13, we have

$$
\begin{aligned}
& \left\|x_{n_{j}}-\left(x^{*}-\xi B x^{*}\right)\right\| \\
& \leq\left\|x_{n_{j}+1}-x_{n_{j}}\right\| \\
& +\| \alpha_{n_{j}} u+\beta_{n_{j}} x_{n_{j}}+\left[\left(1-\beta_{n_{j}}\right) I-\alpha_{n_{j}} A\right] \\
& \quad \times W_{n_{j}}(I-\xi B) F_{r_{n_{j}}} G_{r_{n_{j}}} x_{n_{j}}-\left(x^{*}-\xi B x^{*}\right) \| \\
& \leq\left\|x_{n_{j}+1}-x_{n_{j}}\right\|+\beta_{n_{j}}\left\|x_{n_{j}}-\left(x^{*}-\xi B x^{*}\right)\right\|+\left(1-\beta_{n_{j}}\right) \\
& \times\left(\| W_{n_{j}}(I-\xi B) F_{r_{n_{j}}} G_{r_{n_{j}}} x_{n_{j}}\right. \\
& \quad-(I-\xi B) F_{r_{n_{j}}} G_{r_{n_{j}}} x_{n_{j}} \| \\
& +\|(I-\xi B) F_{r_{n_{j}}} G_{r_{n_{j}}} x_{n_{j}} \\
& \left.\quad-\left(x^{*}-\xi B x^{*}\right) \|\right) \\
& +\alpha_{n_{j}}\left\|u-A W W_{n_{j}}(I-\xi B) F_{r_{n_{j}}} G_{r_{n_{j}}} x_{n_{j}}\right\|
\end{aligned}
$$

$$
\begin{aligned}
& \leq\left\|x_{n_{j}+1}-x_{n_{j}}\right\|+\beta_{n_{j}}\left\|x_{n_{j}}-\left(x^{*}-\xi B x^{*}\right)\right\|+\left(1-\beta_{n_{j}}\right) \\
& \times\left(\| W_{n_{j}}(I-\xi B) F_{r_{n_{j}}} G_{r_{n_{j}}} x_{n_{j}}\right. \\
& -(I-\xi B) F_{r_{n_{j}}} G_{r_{n_{j}}} x_{n_{j}} \| \\
& \left.+\left\|F_{r_{n_{j}}} G_{r_{n_{j}}} x_{n_{j}}-x^{*}\right\|\right) \\
& +\alpha_{n_{j}}\left\|u-A W_{n_{j}}(I-\xi B) F_{r_{n_{j}}} G_{r_{n_{j}}} x_{n_{j}}\right\| \\
& \leq\left\|x_{n_{j}+1}-x_{n_{j}}\right\|+\beta_{n_{j}}\left\|x_{n_{j}}-\left(x^{*}-\xi B x^{*}\right)\right\|+\left(1-\beta_{n_{j}}\right) \\
& \times\left(\| W_{n_{j}}(I-\xi B) F_{r_{n_{j}}} G_{r_{n_{j}}} x_{n_{j}}\right. \\
& -(I-\xi B) F_{r_{n_{j}}} G_{r_{n_{j}}} x_{n_{j}} \| \\
& +\left\|F_{r_{n_{j}}} G_{r_{n_{j}}} x_{n_{j}}-F_{r_{n_{j}}} G_{r_{0}} x^{*}\right\| \\
& \left.+\left\|F_{r_{n_{j}}} G_{r_{0}} x^{*}-F_{r_{0}} G_{r_{0}} x^{*}\right\|\right) \\
& +\alpha_{n_{j}}\left\|u-A W_{n_{j}}(I-\xi B) F_{r_{n_{j}}} G_{r_{n_{j}}} x_{n_{j}}\right\| \\
& \leq\left\|x_{n_{j}+1}-x_{n_{j}}\right\|+\beta_{n_{j}}\left\|x_{n_{j}}-\left(x^{*}-\xi B x^{*}\right)\right\|+\left(1-\beta_{n_{j}}\right) \\
& \times\left(\| W_{n_{j}}(I-\xi B) F_{r_{n_{j}}} G_{r_{n_{j}}} x_{n_{j}}\right. \\
& -(I-\xi B) F_{r_{n_{j}}} G_{r_{n_{j}}} x_{n_{j}} \| \\
& +\left\|G_{r_{n_{j}}} x_{n_{j}}-G_{r_{0}} x^{*}\right\| \\
& \left.+\left\|F_{r_{n_{j}}} G_{r_{0}} x^{*}-F_{r_{0}} G_{r_{0}} x^{*}\right\|\right) \\
& +\alpha_{n_{j}}\left\|u-A W_{n_{j}}(I-\xi B) F_{r_{n_{j}}} G_{r_{n_{j}}} x_{n_{j}}\right\| .
\end{aligned}
$$

This implies that

$$
\begin{aligned}
\left\|x_{n_{j}}-\left(x^{*}-\xi B x^{*}\right)\right\| & \\
\leq\left\|x_{n_{j}+1}-x_{n_{j}}\right\| & +\beta_{n_{j}}\left\|x_{n_{j}}-\left(x^{*}-\xi B x^{*}\right)\right\| \\
+\left(1-\beta_{n_{j}}\right)(\| & W_{n_{j}}(I-\xi B) F_{r_{n_{j}}} G_{r_{n_{j}}} x_{n_{j}} \\
& -(I-\xi B) F_{r_{n_{j}}} G_{r_{n_{j}}} x_{n_{j}} \| \\
& +\left\|G_{r_{n_{j}}} x_{n_{j}}-G_{r_{n_{j}}} x^{*}\right\|
\end{aligned}
$$




$$
\begin{aligned}
& +\left\|G_{r_{n_{j}}} x^{*}-G_{r_{0}} x^{*}\right\| \\
& \left.+\left\|F_{r_{n_{j}}} G_{r_{0}} x^{*}-F_{r_{0}} G_{r_{0}} x^{*}\right\|\right) \\
& +\alpha_{n_{j}}\left\|u-A W_{n_{j}}(I-\xi B) F_{r_{n_{j}}} G_{r_{n_{j}}} x_{n_{j}}\right\| \\
& \leq\left\|x_{n_{j}+1}-x_{n_{j}}\right\|+\beta_{n_{j}}\left\|x_{n_{j}}-\left(x^{*}-\xi B x^{*}\right)\right\| \\
& +\left(1-\beta_{n_{j}}\right)\left(\| W_{n_{j}}(I-\xi B) F_{r_{n_{j}}} G_{r_{n_{j}}} x_{n_{j}}\right. \\
& -(I-\xi B) F_{r_{n_{j}}} G_{r_{n_{j}}} x_{n_{j}} \| \\
& +\left\|x_{n_{j}}-x^{*}\right\|+\frac{\left|r_{n_{j}}-r_{0}\right|}{r_{0}} \\
& \left.\times\left(\left\|F_{r_{0}} G_{r_{0}} x^{*}\right\|+2\left\|G_{r_{0}} x^{*}\right\|+\left\|x^{*}\right\|\right)\right) \\
& +\alpha_{n_{j}}\left\|u-A W_{n_{j}}(I-\xi B) F_{r_{n_{j}}} G_{r_{n_{j}}} x_{n_{j}}\right\| .
\end{aligned}
$$

Inequality (129) thus gives

$$
\begin{aligned}
& \left\|x_{n_{j}}-\left(x^{*}-\xi B x^{*}\right)\right\| \\
& \leq \quad \frac{1}{1-\beta_{n_{j}}}\left\|x_{n_{j}+1}-x_{n_{j}}\right\| \\
& \quad+\left\|W_{n_{j}}(I-\xi B) F_{r_{n_{j}}} G_{r_{n_{j}}} x_{n_{j}}-(I-\xi B) F_{r_{n_{j}}} G_{r_{n_{j}}} x_{n_{j}}\right\| \\
& \quad+\left\|x_{n_{j}}-x^{*}\right\|+4\left\|x^{*}\right\| \frac{\left|r_{n_{j}}-r_{0}\right|}{r_{0}} \\
& \quad+\frac{\alpha_{n_{j}}}{1-\beta_{n_{j}}}\left\|u-A W_{n_{j}}(I-\xi B) F_{r_{n_{j}}} G_{r_{n_{j}}} x_{n_{j}}\right\| .
\end{aligned}
$$

Thus, we obtain from (130) that

$$
\underset{j \rightarrow \infty}{\limsup }\left\|x_{n_{j}}-\left(x^{*}-\xi B x^{*}\right)\right\| \leq \underset{j \rightarrow \infty}{\limsup }\left\|x_{n_{j}}-x^{*}\right\|,
$$

so that Definition 10 again gives $x^{*}-\xi B x^{*}=x^{*}$ and this implies that $x^{*} \in B^{-1}(0)$. Hence, $x^{*} \in \Omega$.
Now, let $\varepsilon \in(0,1)$ and $x \in H$ be arbitrary, then, using Lemma 4 we have

$$
\begin{aligned}
&\left\|x_{n_{j}}-x^{*}-\varepsilon\left(x-x^{*}\right)\right\|^{2} \\
& \leq\left\|x_{n_{j}}-x^{*}\right\|^{2} \\
& \quad-2 \varepsilon\left\langle x-x^{*}, x_{n_{j}}-x^{*}-\varepsilon\left(x-x^{*}\right)\right\rangle \\
&=\left\|x_{n_{j}}-x^{*}\right\|^{2}+2 \varepsilon\left\langle x-x^{*}, x^{*}+\varepsilon\left(x-x^{*}\right)-x_{n_{j}}\right\rangle .
\end{aligned}
$$

Since $x^{*}$ is the asymptotic center of $\left\{x_{n_{j}}\right\}_{j \geq 1}$, it is easy to see (using Definition 10 and Lemma 14) that

$$
\begin{aligned}
& \underset{j \rightarrow \infty}{\limsup }\left\|x_{n_{j}}-x^{*}\right\|^{2} \\
& \quad \leq \limsup _{j \rightarrow \infty}\left\|x_{n_{j}}-x^{*}-\varepsilon\left(x-x^{*}\right)\right\|^{2} \\
& \leq \underset{j \rightarrow \infty}{\leq \limsup _{j \rightarrow \infty}\left\|x_{n_{j}}-x^{*}\right\|^{2}} \\
& \quad+2 \varepsilon \limsup _{j \rightarrow \infty}\left\langle x-x^{*}, x^{*}+\varepsilon\left(x-x^{*}\right)-x_{n_{j}}\right\rangle .
\end{aligned}
$$

Thus,

$$
0 \leq \limsup _{j \rightarrow \infty}\left\langle x-x^{*}, x^{*}+\varepsilon\left(x-x^{*}\right)-x_{n_{j}}\right\rangle,
$$

But,

$$
\begin{aligned}
\left\langle x-x^{*}, x^{*}-x_{n_{j}}\right\rangle= & \left\langle x-x^{*}, x^{*}+\varepsilon\left(x-x^{*}\right)-x_{n_{j}}\right\rangle \\
& -\varepsilon\left\|x-x^{*}\right\|^{2}
\end{aligned}
$$

So,

$$
\begin{aligned}
& \limsup _{j \rightarrow \infty}\left\langle x-x^{*}, x^{*}-x_{n_{j}}\right\rangle \\
& \quad=\limsup _{j \rightarrow \infty}\left(\left\langle x-x^{*}, x^{*}+\varepsilon\left(x-x^{*}\right)-x_{n_{j}}\right\rangle-\varepsilon\left\|x-x^{*}\right\|^{2}\right) \\
& \quad=\limsup _{j \rightarrow \infty}\left\langle x-x^{*}, x^{*}+\varepsilon\left(x-x^{*}\right)-x_{n_{j}}\right\rangle-\varepsilon\left\|x-x^{*}\right\|^{2} \\
& \geq-\varepsilon\left\|x-x^{*}\right\|^{2} .
\end{aligned}
$$

Thus, since $\varepsilon \in(0,1)$ is arbitrary, we obtain from (136) that

$$
\limsup _{j \rightarrow \infty}\left\langle x-x^{*}, x^{*}-x_{n_{j}}\right\rangle \geq 0 \quad \forall x \in H \text {. }
$$


In particular, for $x=x^{*}+u-A x^{\prime} \in H$ and using the fact that $x^{*} \in \Omega$, we obtain from (137) that

$$
\begin{aligned}
0 & \leq \limsup _{j \rightarrow \infty}\left\langle u-A x^{\prime}, x^{*}-x_{n_{j}}\right\rangle \\
& \leq\left\langle u-A x^{\prime}, x^{*}-x^{\prime}\right\rangle+\limsup _{j \rightarrow \infty}\left\langle u-A x^{\prime}, x^{\prime}-x_{n_{j}}\right\rangle \\
& =\left\langle u-A x^{\prime}, x^{*}-x^{\prime}\right\rangle+\lim _{j \rightarrow \infty}\left\langle u-A x^{\prime}, x^{\prime}-x_{n_{j}}\right\rangle \\
& \leq \lim _{j \rightarrow \infty}\left\langle u-A x^{\prime}, x^{\prime}-x_{n_{j}}\right\rangle .
\end{aligned}
$$

This implies that

$$
\lim _{j \rightarrow \infty}\left\langle u-A x^{\prime}, x_{n_{j}}-x^{\prime}\right\rangle \leq 0 .
$$

Hence,

$$
\limsup _{n \rightarrow \infty}\left\langle u-A x^{\prime}, x_{n}-x^{\prime}\right\rangle=\lim _{j \rightarrow \infty}\left\langle u-A x^{\prime}, x_{n_{j}}-x^{\prime}\right\rangle \leq 0 .
$$

Theorem 22. Let $\left\{x_{n}\right\}_{n \geq 1}$ be given by (74). Suppose that the conditins of Remark 16 are satisfied, then $\left\{x_{n}\right\}_{n \geq 1}$ converges strongly to $x^{\prime} \in \Omega$ which is a unique solution of the variational inequality (75).

Proof. From the recursion formula (74) and Lemma 4, we have

$$
\begin{aligned}
\left\|x_{n+1}-x^{\prime}\right\|^{2} & \\
=\| \alpha_{n} u+\left[\left(1-\beta_{n}\right) I-\alpha_{n} A\right] & \times W_{n}(I-\xi B) F_{r_{n}} G_{r_{n}} x_{n}+\beta_{n} x_{n}-x^{\prime} \|^{2} \\
=\| & {\left[\left(1-\beta_{n}\right) I-\alpha_{n} A\right] } \\
& \times\left(W_{n}(I-\xi B) F_{r_{n}} G_{r_{n}} x_{n}-x^{\prime}\right) \\
+ & \beta_{n}\left(x_{n}-x^{\prime}\right)+\alpha_{n}\left(u-A x^{\prime}\right) \|^{2} \\
\leq \| & {\left[\left(1-\beta_{n}\right) I-\alpha_{n} A\right] } \\
& \times\left(W_{n}(I-\xi B) F_{r_{n}} G_{r_{n}} x_{n}-x^{\prime}\right)+\beta_{n}\left(x_{n}-x^{\prime}\right) \|^{2} \\
+ & 2 \alpha_{n}\left\langle u-A x^{\prime}, x_{n+1}-x^{\prime}\right\rangle
\end{aligned}
$$

$=\|\left[\left(1-\beta_{n}\right) I-\alpha_{n} A\right]$

$$
\times\left(W_{n}(I-\xi B) F_{r_{n}} G_{r_{n}} x_{n}-x^{\prime}\right)+\beta_{n}\left(x_{n}-x^{\prime}\right) \|^{2}
$$$$
+2 \alpha_{n}\left\langle u-A x^{\prime},\left(1-\beta_{n}\right)\right.
$$$$
\times\left(W_{n}(I-\xi B) F_{r_{n}} G_{r_{n}} x_{n}-x^{\prime}\right)
$$$$
-\alpha_{n} A\left(W_{n}(I-\xi B) F_{r_{n}} G_{r_{n}} x_{n}-x^{\prime}\right)
$$$$
\left.+\alpha_{n}\left(u-A x^{\prime}\right)+\beta_{n}\left(x_{n}-x^{\prime}\right)\right\rangle
$$$$
=\|\left[\left(1-\beta_{n}\right) I-\alpha_{n} A\right]
$$

$$
\begin{aligned}
& \times\left(W_{n}(I-\xi B) F_{r_{n}} G_{r_{n}} x_{n}-x^{\prime}\right)+\beta_{n}\left(x_{n}-x^{\prime}\right) \|^{2} \\
+ & 2 \alpha_{n}^{2}\left\|u-A x^{\prime}\right\|^{2}+2 \alpha_{n}\left(1-\beta_{n}\right) \\
\times & \left\langle u-A x^{\prime}, W_{n}(I-\xi B) F_{r_{n}} G_{r_{n}} x_{n}-x^{\prime}\right\rangle \\
- & 2 \alpha_{n}^{2}\left\langle u-A x^{\prime}, A\left(W_{n}(I-\xi B) F_{r_{n}} G_{r_{n}} x_{n}-x^{\prime}\right)\right\rangle \\
+ & 2 \alpha_{n} \beta_{n}\left\langle u-A x^{\prime}, x_{n}-x^{\prime}\right\rangle
\end{aligned}
$$$$
\leq\left(1-\beta_{n}-\alpha_{n} \gamma+\beta_{n}\right)^{2}\left\|x_{n}-x^{\prime}\right\|^{2}
$$$$
+2 \alpha_{n}^{2}\left\|u-A x^{\prime}\right\|^{2}+2 \alpha_{n}\left(1-\beta_{n}\right)
$$$$
\times\left\langle u-A x^{\prime}, W_{n}(I-\xi B) F_{r_{n}} G_{r_{n}} x_{n}-x_{n}\right\rangle
$$$$
+2 \alpha_{n}\left(1-\beta_{n}\right)\left\langle u-A x^{\prime}, x_{n}-x^{\prime}\right\rangle
$$$$
-2 \alpha_{n}^{2}\left\langle u-A x^{\prime}, A\left(W_{n}(I-\xi B) F_{r_{n}} G_{r_{n}} x_{n}-x^{\prime}\right)\right\rangle
$$$$
+2 \beta_{n} \alpha_{n}\left\langle u-A x^{\prime}, x_{n}-x^{\prime}\right\rangle
$$$$
=\left[1-2 \alpha_{n} \gamma\right]\left\|x_{n}-x^{\prime}\right\|^{2}
$$$$
+\alpha_{n}^{2} \gamma^{2}\left\|x_{n}-x^{\prime}\right\|^{2}+2 \alpha_{n}^{2}\left\|u-A x^{\prime}\right\|^{2}+2 \alpha_{n}\left(1-\beta_{n}\right)
$$$$
\times\left\langle u-A x^{\prime}, W_{n}(I-\xi B) F_{r_{n}} G_{r_{n}} x_{n}-x_{n}\right\rangle
$$$$
-2 \alpha_{n}^{2}\left\langle u-A x^{\prime}, A W_{n}(I-\xi B) F_{r_{n}} G_{r_{n}} x_{n}-x^{\prime}\right\rangle
$$$$
+2 \alpha_{n}\left\langle u-A x^{\prime}, x_{n}-x^{\prime}\right\rangle
$$$$
\leq\left[1-2 \alpha_{n} \gamma\right]\left\|x_{n}-x^{\prime}\right\|^{2}
$$$$
+\alpha_{n}\left(\alpha_{n}+\left\|W_{n}(I-\xi B) F_{r_{n}} G_{r_{n}} x_{n}-x_{n}\right\|\right) M_{4}
$$$$
+2 \alpha_{n}\left\langle u-A x^{\prime}, x_{n}-x^{\prime}\right\rangle \text {, }
$$ 
for some real constant $M_{4}>0$. Thus,

$$
\left\|x_{n+1}-x^{\prime}\right\|^{2} \leq\left(1-\delta_{n}\right)\left\|x_{n}-x^{\prime}\right\|^{2}+\sigma_{n},
$$

where $\delta_{n}:=2 \alpha_{n} \gamma$ and $\sigma_{n}:=\alpha_{n}\left(\alpha_{n}+\| W_{n}(I-\xi B) F_{r_{n}} G_{r_{n}} x_{n}-\right.$ $\left.x_{n} \|\right) M_{4}+2 \alpha_{n}\left\langle u-A x^{\prime}, x_{n}-x^{\prime}\right\rangle$. It is easy to see that $\limsup _{n \rightarrow \infty}\left(\sigma_{n} / \delta_{n}\right) \leq 0$ (using (93), Lemma 21 and the fact that $\lim _{n \rightarrow \infty} \alpha_{n}=0$ ). Hence, we obtain by Lemma 5 that the sequence $\left\{x_{n}\right\}_{n \geq 1}$ converges strongly to $x^{\prime} \in \Omega$ which is a unique solution of (75).

Remark 23. Let $f: H \rightarrow H$ be a strict contraction, then our method of proof easily carries over to the iteration method $\left\{x_{n}\right\}_{n \geq 1}$ given by $x_{1} \in H$,

$$
\begin{aligned}
x_{n+1}= & \alpha_{n} \gamma f\left(x_{n}\right)+\beta x_{n} \\
& +\left(\left(1-\beta_{n}\right) I-\alpha_{n} A\right) W_{n}(I-\xi B) F_{r_{n}} G_{r_{n}} x_{n},
\end{aligned}
$$

making use of the fact that $f$ is a contraction. Thus, we obatin the following theorem.

Theorem 24. Let $\left\{x_{n}\right\}_{n \geq 1}$ be given by (143). Suppose that the conditions of Remark 16 are satisfied, then $\left\{x_{n}\right\}_{n \geq 1}$ converges strongly to $x^{\prime} \in \Omega$ which is a unique solution of the variational inequality

$$
\begin{gathered}
\left\langle(A-\gamma f) x^{\prime}, y-x^{\prime}\right\rangle \geq 0, \\
\forall y \in \Omega \Longleftrightarrow x^{\prime}=P_{\Omega}(I-A+\gamma f) x^{\prime},
\end{gathered}
$$

which is the optimality condition for the minimization problem $\min _{x \in \Omega}(1 / 2)\langle A x, x\rangle-h(x)$, where $h$ is a potential function for $\gamma f$ (i.e., $h^{\prime}(x)=\gamma f(x)$ for all $x \in H$ ), and one recalls that $\Omega=\operatorname{Fix}\left(T^{\prime}\right) \cap \operatorname{GMEP}(f, \Phi, \Theta) \cap B^{-1}(0) \cap \bigcap_{j=1}^{\infty} \operatorname{Fix}\left(T_{j}\right)$.

Remark 25. Our theorems extend and unify most of the results that have been proved for the class of nonlinear operators studied in this paper. In particular, it is easy to see that Theorem 24 extends, improves, generalizes, and unifies the corresponding results of Yao et al. [32], Colao and Marino [33], and Chamnarnpan and Kuman [34]. Furthermore, the setting of our theorems corrects the problem of well definedness which might arise in the results of Chamnarnpan and Kuman [34], and our method of proof is of independent interest.

\section{References}

[1] C. E. Chidume, "Geometric properties of banach spaces and nonlinear iterations ABC," Lecture Notes in Mathematics, vol. 1965, pp. 1-325, 2009.

[2] T. Kato, "Nonlinear semigroups and evolution equations," Journal of the Mathematical Society of Japan, vol. 19, pp. 508-520, 1967.

[3] E. U. Ofoedu and H. Zegeye, "Further investigation on iteration processes for pseudocontractive mappings with application," Nonlinear Analysis: Theory, Methods \& Applications, vol. 75, Article ID 153162, 2012.
[4] E. Blum and W. Oettli, "From optimization and variational inequalities to equilibrum problems," The Mathematics Student, vol. 63, no. 1-4, pp. 123-145, 1994.

[5] P. Katchang, T. Jitpeera, and P. Kumam, "Strong convergence theorems for solving generalized mixed equilibrium problems and general system of variational inequalities by the hybrid method," Nonlinear Analysis, vol. 4, no. 4, pp. 838-852, 2010.

[6] H. Zegeye, E. U. Ofoedu, and N. Shahzad, "Convergence theorems for equilibrium problem, variational inequality problem and countably infinite relatively quasi-nonexpansive mappings," Applied Mathematics and Computation, vol. 216, no. 12, pp. 3439-3449, 2010.

[7] C. E. Chidume and E. U. Ofoedu, "A new iteration process for finite families of generalized Lipschitz pseudo-contractive and generalized Lipschitz accretive mappings," Nonlinear Analysis, Theory, Methods and Applications, vol. 69, no. 4, pp. 1200-1207, 2008.

[8] C. E. Chidume and E. U. Ofoedu, "Approximation of common fixed points for finite families of total asymptotically nonexpansive mappings," Journal of Mathematical Analysis and Applications, vol. 333, no. 1, pp. 128-141, 2007.

[9] S. Ishikawa, "Fixed point by a new iteration method," Proceedings of the American Mathematical Society, vol. 44, pp. 147-150, 1974.

[10] W. R. Mann, "Mean value methods in iteration," Proceedings of the American Mathematical Society, vol. 4, pp. 506-510, 1953.

[11] G. Marino and H. K. Xu, "A general iterative method for nonexpansive mappings in Hilbert spaces," Journal of Mathematical Analysis and Applications, vol. 318, no. 1, pp. 43-52, 2006.

[12] R. E. Megginson, An Introduction to Banach Space Theory, Springer, New York, NY, USA, 1998.

[13] A. Moudafi, "Viscosity approximation methods for fixed-points problems," Journal of Mathematical Analysis and Applications, vol. 241, no. 1, pp. 46-55, 2000.

[14] E. U. Ofoedu, "Iterative approximation of a common zero of a countably infinite family of $\mathrm{m}$-accretive operators in Banach spaces," Fixed Point Theory and Applications, vol. 2008, Article ID 325792, 2008.

[15] E. U. Ofoedu and Y. Shehu, "Iterative construction of a common fixed point of finite families of nonlinear mappings," Journal of Applied Analysis, vol. 16, no. 1, pp. 59-77, 2010.

[16] E. U. Ofoedu, Y. Shehu, and J. N. Ezeora, "Solution by iteration of nonlinear variational inequalities involving finite family of asymptotically nonexpansive mappings and monotone mappings," Panamerican Mathematical Journal, vol. 18, no. 4, pp. 61-75, 2008.

[17] S. Reich, "Weak convergence theorems for nonexpansive mappings in Banach spaces," Journal of Mathematical Analysis and Applications, vol. 67, no. 2, pp. 274-276, 1979.

[18] S. Reich, "Extension problems for accretive sets in Banach spaces," Journal of Functional Analysis, vol. 26, no. 4, pp. 378-395, 1977.

[19] S. Reich, "Strong convergence theorems for resolvents of accretive operators in Banach spaces," Journal of Mathematical Analysis and Applications, vol. 75, no. 1, pp. 287-292, 1980.

[20] B. E. Rhoades, "Fixed point iterations for certain nonlinear mappings," Journal of Mathematical Analysis and Applications, vol. 183, no. 1, pp. 118-120, 1994.

[21] R. T. Rockafellar, "Monotone operators and proximal point algorithm," SIAM Journal on Control and Optimization, vol. 14, no. 5, pp. 877-898, 1976. 
[22] D. Sahani and R. K. Bose, "On asymptotic centres and fixed point theorems for set-valued nonexpansive mappings," Indian Journal of Pure and Applied Mathematics, vol. 15, no. 9, pp. 991-997, 1984.

[23] J. Schu, "Iterative construction of fixed points of asymptotically nonexpansive mappings," Journal of Mathematical Analysis and Applications, vol. 158, no. 2, pp. 407-413, 1991.

[24] J. Schu, "Weak and strong convergence of fiexd points of asymptotically nonexpansive mappings," Bulletin of the Australian Mathematical Society, vol. 43, pp. 153-159, 1991.

[25] H. H. Bauschke, "The approximation of fixed points of compositions of nonexpansive mappings in Hilbert space," Journal of Mathematical Analysis and Applications, vol. 202, no. 1, pp. 150-159, 1996.

[26] B. Halpern, "Fixed points of nonexpanding maps," Bulletin of the American Mathematical Society, vol. 73, pp. 957-961, 1967.

[27] P. L. Lions, "Approximation de points fixes de contractions," Comptes Rendus de l'Académie des Sciences, vol. 284, pp. 1357-1359, 1997.

[28] R. Wittmann, "Approximation of fixed points of nonexpansive mappings," Archiv der Mathematik, vol. 58, no. 5, pp. 486-491, 1992.

[29] I. Yamada, "The hybrid steepest decent for the variational inequality problems over the itersection of fixed points sets of nonexpansive mapping," in Inherently Parallel Algorithme in Feasibility and Optimization and their Application, D. Butnariu, Y. Censor, and S. Reich, Eds., pp. 473-504, Elsevier, New York, NY, USA, 2001.

[30] M. Tian, "A general iterative algorithm for nonexpansive mappings in Hilbert spaces," Nonlinear Analysis, vol. 73, no. 3, pp. 689-694, 2010.

[31] K. Shimoji and W. Takahashi, "Strong convergence to common fixed points of infinite nonexpansive mappings and applications," Taiwanese Journal of Mathematics, vol. 5, no. 2, pp. 387-404, 2001.

[32] Y. Yao, Y. C. Liou, and R. Chen, "A general iterative method for an infinite family of nonexpansive mappings," Nonlinear Analysis, Theory, Methods and Applications, vol. 69, no. 5-6, pp. 1644-1654, 2008.

[33] V. Colao and G. Marino, "Strong convergence for a minimization problem on points of equilibrium and common fixed points of an infinite family of nonexpansive mappings," Nonlinear Analysis, Theory, Methods and Applications, vol. 73, no. 11, pp. 3513-3524, 2010.

[34] T. Chamnarnpan and P. Kuman, "A new iterative method for a common solution of fixed points for pseudo-contractive mappings and variational inequalities," Fixed Point Theory and Applications, vol. 2012, 67 pages, 2012.

[35] V. Berinde, Iterative approximation of fixed points, Editura Efemeride Baia Mare, 2002.

[36] H. K. Xu, "Iterative algorithms for nonlinear operators," Journal of the London Mathematical Society, vol. 66, no. 1, pp. 240-256, 2002.

[37] H. K. Xu, "An iterative approach to quadratic optimization," Journal of Optimization Theory and Applications, vol. 116, no. 3, pp. 659-678, 2003.

[38] T. Suzuki, "Strong convergence of Krasnoselskii and Mann's type sequences for one-parameter nonexpansive semigroups without Bochner integrals," Journal of Mathematical Analysis and Applications, vol. 305, no. 1, pp. 227-239, 2005.
[39] H. Zegeye, "An iterativee approximation method for a common fixed point of two pseudo-contractive mappings," ISRN Mathematical Analysis, vol. 2011, Article ID 621901, 14 pages, 2011.

[40] M. Edelstein, "The construction of an asymptotic center with a fixed-point property," Bulletin of the American Mathematical Society, vol. 78, no. 2, pp. 206-208, 1972. 


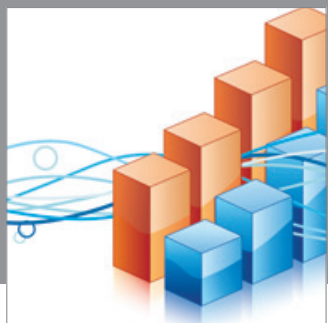

Advances in

Operations Research

mansans

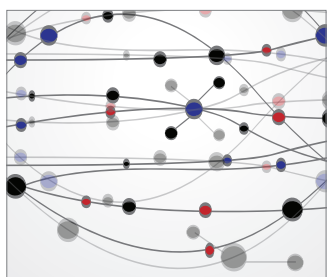

The Scientific World Journal
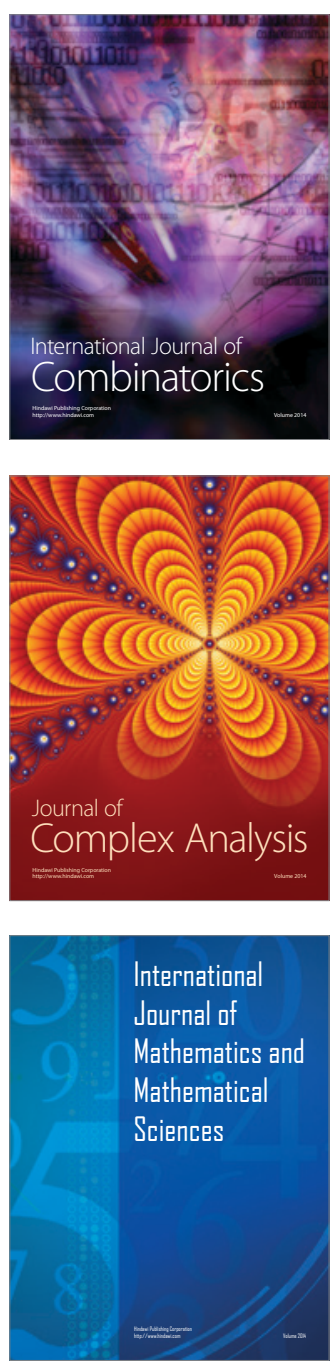
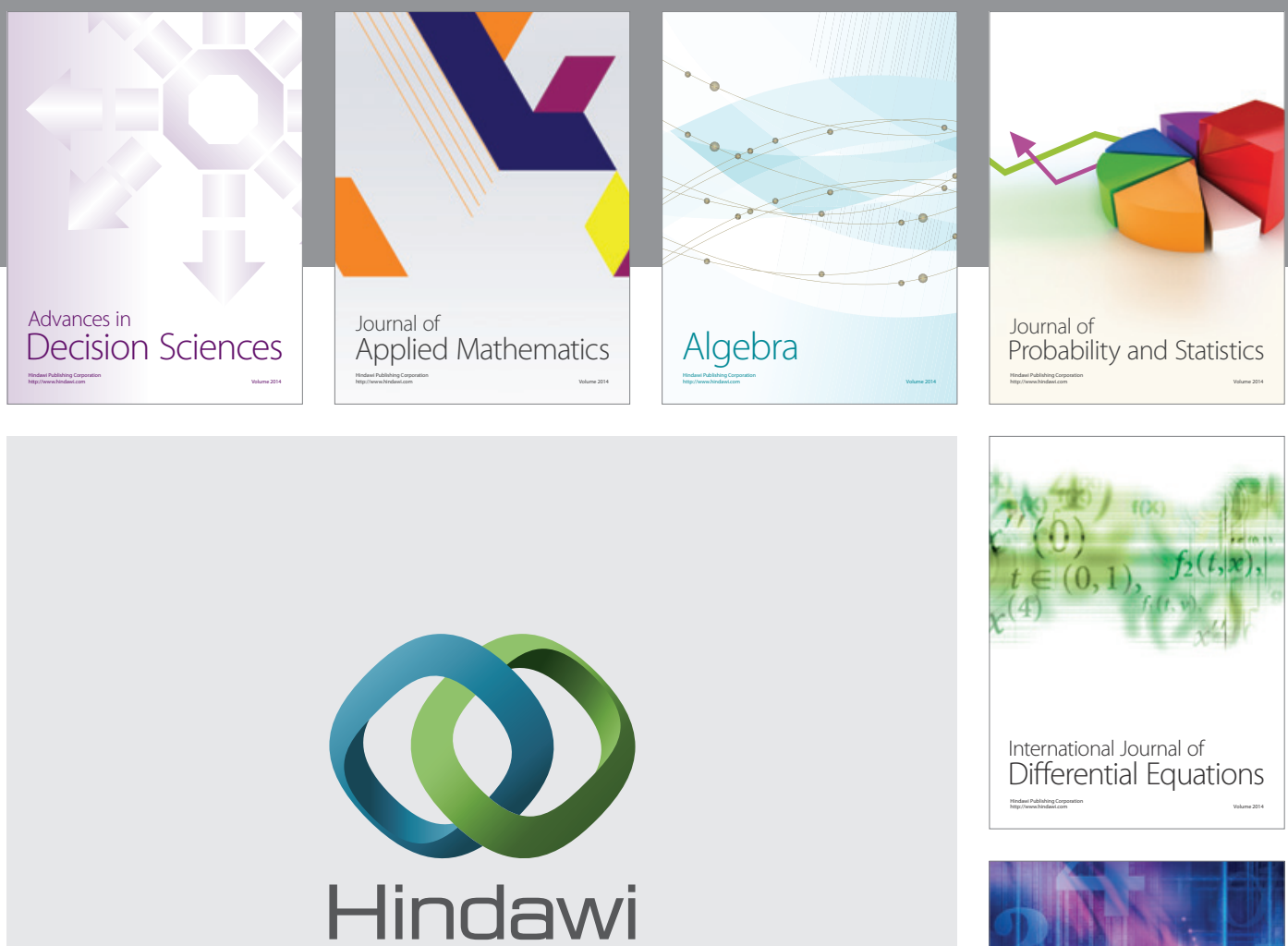

Submit your manuscripts at http://www.hindawi.com
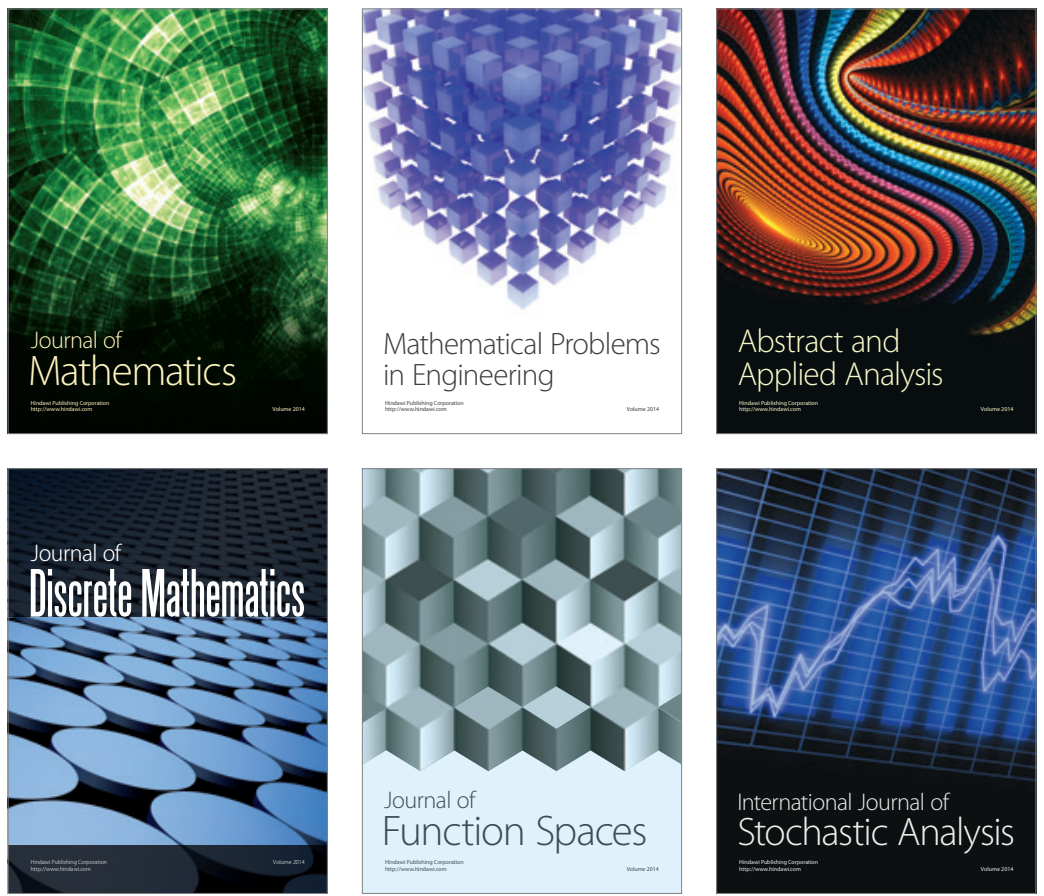

Journal of

Function Spaces

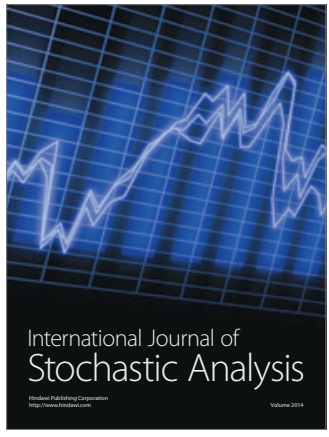

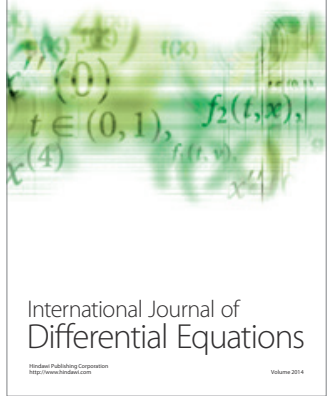
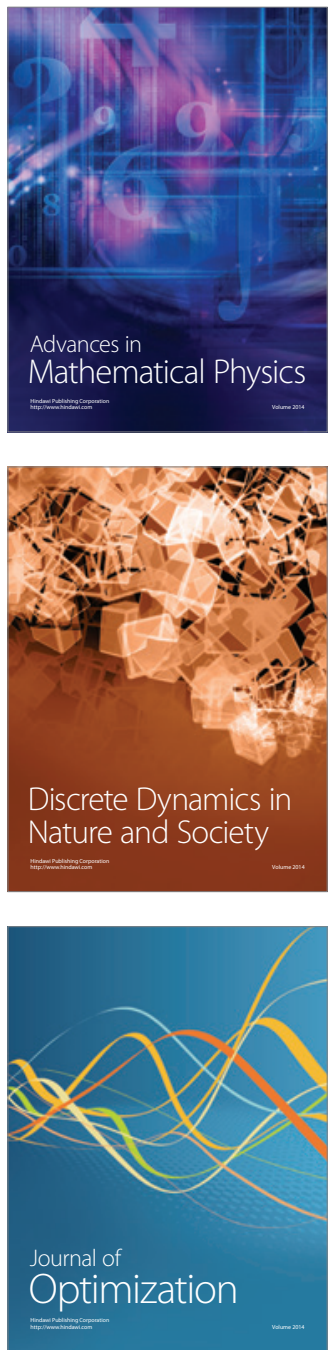